\title{
Spectral footprints of impurity scattering in graphene nanoribbons
}

\author{
Anders Bergvall and Tomas Löfwander \\ Department of Microtechnology and Nanoscience-MC2, Chalmers University of Technology, SE-412 96 Göteborg, Sweden
}

(Received 22 December 2012; published 22 May 2013)

\begin{abstract}
We report a detailed investigation of the interplay between size quantization and local scattering centers in graphene nanoribbons, as seen in the local density of states. The spectral signatures, obtained after Fourier transformation of the local density of states, include characteristic peaks that can be related to the transverse modes of the nanoribbon. In armchair ribbons, the Fourier transformed density of states of one of the two inequivalent sublattices takes a form similar to that of a quantum channel in a two-dimensional electron gas, modified according to the differences in band structure. After addition of the second sublattice contribution, a characteristic modulation of the pattern due to superposition is obtained, similar to what has been obtained in spectra due to single impurity scattering in large-area graphene. We present analytic results for the electron propagator in armchair nanoribbons in the Dirac approximation, including a single scattering center within a $T$-matrix formulation. For comparison, we have extended the investigation with numerics obtained with an atomistic recursive Green's function approach. The spectral signatures of the atomistic approach include the effects of trigonal warping. The impurity induced oscillations in the local density of states are not decaying at large distance in few-mode nanoribbons.
\end{abstract}

DOI: 10.1103/PhysRevB.87.205431

PACS number(s): 73.22.-f, 73.22.Pr

\section{INTRODUCTION}

In graphene, scattering centers such as impurities, defects, adatoms, and substrate inhomogeneities greatly influence the local electronic properties. ${ }^{1}$ In some samples, the material quality is so high that a single or a few such scattering centers can influence the whole device. This may degrade device function, but can also be taken advantage of by making various sensing devices. ${ }^{2,3}$ Great attention has therefore been focused on understanding the influence of scattering on the electronic properties of graphene. ${ }^{4}$

In this context, the scanning tunneling microscope (STM) is becoming of increasing importance. ${ }^{5,6}$ By utilizing its various modes of operation, the STM can be used to map out topography, local density of states, local charge density, and more. In this way, a variety of properties of graphene have been revealed. A few examples include perturbations in the local density of states around impurities ${ }^{7,8}$ or near step edges in the substrate, ${ }^{9}$ charge puddle formation caused by molecules trapped between graphene flakes and the $\mathrm{SiO}_{2}$ substrate, ${ }^{10}$ and resistance caused by steps ${ }^{11,12}$ in the substrate or multilayer regions ${ }^{13}$ in epitaxial graphene on silicon-carbide.

At the same time, encouraging progress has been achieved with fabrication of graphene nanostructures. Topdown approaches include nanolithography, ${ }^{14}$ scanning probe methods, ${ }^{15}$ etching with metal nanoparticles along certain crystal directions, ${ }^{16}$ and utilization of the transmission electron microscope (TEM) to simultaneously image and sculpture graphene. ${ }^{17}$ A bottom-up approach based on chemical synthesis has also been demonstrated. ${ }^{18}$ Another approach involves unzipping of carbon nanotubes. ${ }^{19}$ With that method, the theoretically predicted zero-energy (midgap) edge states of nanoribbons with zigzag edges ${ }^{20,21}$ were directly mapped out by scanning tunneling spectroscopy (STS). ${ }^{22}$ Theory also predicts that by controlling the width and edges of nanoribbons, a band gap can be opened up at the Dirac point through quantum confinement (see the review ${ }^{23}$ ). With further progress it may soon become possible to study in much greater detail the interplay between quantum confinement and impurity scattering in graphene nanoribbons.

Many theoretical studies of graphene nanoribbons have been reported in the literature, see the collection of review articles in Ref. 24. The effect of impurity scattering and the effects of edge disorder on electron transport have been reported in several numerical works. In an effort to simulate the typical experimental situation, random disorder is included and the scaling behavior of resistivity with length of the ribbon is studied, revealing different transport regimes depending on ribbon width and disorder properties. Here, we go back to the well-defined problem of a single impurity in order to study in detail the effects on the Fourier transformed local density of states (FT-LDOS).

In this paper we present results for the spectral signatures of a local scattering center in graphene, taking into account quantum confinement in a nanoribbon geometry. This study generalizes the consideration of FT-LDOS of a single impurity in bulk graphene $e^{25,26}$ to the case of nanoribbons. We focus the analytic analysis on armchair ribbons in the Dirac approximation (linearization around the $K$ points in the graphene band structure), for which the wave functions and propagators for clean ribbons are known, and solve the impurity problem in a $T$-matrix formulation. Thereby we obtain the electron propagator for an armchair nanoribbon including the effects of a local scattering center. The FT-LDOS is then obtained and explained in terms of scattering processes of Dirac quasiparticles confined in the ribbon. We extend the analysis to an atomistic tight-binding model of graphene, utilizing a numerical recursive Green's function approach. The main effect of going beyond the Dirac approximation is trigonal warping, which shows up as a triangular distortion of the FT-LDOS patterns.

For comparison we include an analysis of the FT-LDOS in a quantum ribbon in a two-dimensional electron gas (2DEG). Many features of the FT-LDOS patterns in graphene ribbons can be understood from the somewhat simpler case of a 2DEG, 
and the new features special for graphene can be highlighted. These include a more complicated band structure due to the two inequivalent $K$ points, trigonal warping, as well as interference effects due to the bipartite lattice of graphene.

The outline of the paper is as follows. In Sec. II we discuss the Fourier transform STS method and illustrate the basic scattering processes at play in a nanoribbon. In Sec. III we present results for the FT-LDOS in a 2DEG quantum channel. In Sec. IV we report our results for the FT-LDOS in an armchair graphene nanoribbon within the Dirac approximation and compare with the 2DEG case. In Sec. V we present results of numerical simulations of a tight-binding model, including also zigzag nanoribbons as well as effects of edge disorder on the FT-LDOS. In Sec. VI we summarize the paper and give some conclusions and an outlook. Most technical results of the analytic analysis have been collected in the appendixes.

\section{FOURIER TRANSFORM SCANNING TUNNELING SPECTROSCOPY}

A scattering center induces a perturbation of the local density of states in its vicinity. For elastic scattering, the impurity scatters electrons between states $\vec{k}_{1} \rightarrow \vec{k}_{2}$ with $\epsilon_{\vec{k}_{1}}=\epsilon_{\vec{k}_{2}}$, i.e. on a contour of constant energy $E$. This leads to interference and a wave pattern in the local density of states near the impurity with wave vectors $\vec{q}=\vec{k}_{2}-\vec{k}_{1}$. After Fourier transformation of the local density of states $\rho(\vec{r}, E) \rightarrow \mathcal{N}(\vec{q}, E)$, the wave vectors of the interference pattern are highlighted. The resulting pattern in $\mathcal{N}(\vec{q}, E)$ can then be used to infer the band dispersion $\epsilon_{\vec{k}}$. For instance, this has been done for metal surfaces. ${ }^{27}$ This method has also become a valuable tool for probing the properties of high- $T_{c}$ superconductors. ${ }^{28}$

It is worth mentioning that, neglecting electron-electron interactions, the interference patterns in the local density of states discussed above are related to the Friedel oscillations in the electron density $n(\vec{r})$ through integration over energy including the Fermi-Dirac distribution function, $n(\vec{r})=$ $-e \int \rho(\vec{r}, E) f(E) d E$.

By using the STM, the local density of states can be extracted as function of energy by applying a finite voltage between tip and sample, i.e., by employing STS. By combining Fourier transformation with STS, the band dispersion can be studied in the vicinity of the Fermi energy. This method has therefore become a valuable spectroscopic tool sometimes called Fourier transform scanning tunneling spectroscopy (FT-STS). In graphene, the Fermi energy itself is tunable by a back gate voltage on the substrate that graphene is resting on. Thereby, FT-STS is potentially a valuable tool for studies of graphene. Indeed, experiment reproduces the graphene band structure. 8

STS bears similarities with angle-resolved photoemission spectroscopy (ARPES). STS is ideal for spatially inhomogeneous systems, while ARPES relies on large-area spatially homogeneous samples. Indeed, STS measures the spatially resolved spectral function, i.e., local density of states $\rho(\vec{r} ; E)$, while ARPES measures the momentum-space spectral function $A(\vec{k} ; E)$. By generalizing STS to FT-STS, i.e., Fourier transforming $\rho(\vec{r} ; E) \rightarrow \mathcal{N}(\vec{q} ; E)$, a spectroscopy has been introduced that can be used to study materials, although we should remember that $\mathcal{N}(\vec{q} ; E)$ is not equal to $A(\vec{q} ; E)$.

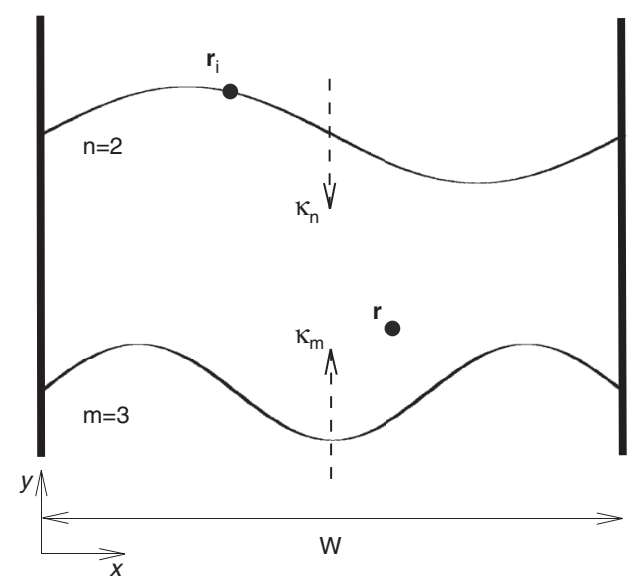

FIG. 1. An electron quasiparticle initially in mode $m$, with longitudinal wave vector $\kappa_{m}$ may be backscattered by an impurity at $\vec{r}_{i}$ into mode $n$. The local density of states at $\vec{r}$ is changed due to interference of the initial and final waves. This leads to an interference pattern around $\vec{r}_{i}$.

One advantage of FT-STS is the possibility to study nanoscale systems with high spatial resolution. In this paper we investigate the consequences of quantum confinement on impurity scattering in graphene, as seen in FT-STS.

In Fig. 1 we display a cartoon of a typical scattering process that contributes to the correction to the local density of states in a quantum ribbon with one impurity. For simplicity we here discuss the situation in a 2DEG quantum channel. Quasiparticles occupying for instance mode $m$, propagating in the positive $y$ direction with wave number $\kappa_{m}$, passes the probing position $\vec{r}=(x, y)$, after which they can be backscattered by the impurity at $\vec{r}_{i}$ into mode $n$ with wave number $\kappa_{n}$ and propagate back to the probing position $\vec{r}$. In this example we neglect evanescent modes for simplicity. The contribution to the full propagator from this scattering event will be proportional to the free propagators before and after scattering and the potential strength $\gamma$,

$$
\begin{aligned}
\tilde{G}_{n m}(\vec{r}, \vec{r} ; E)= & -i \frac{\mu}{\hbar^{2}} \frac{e^{i \kappa_{n}\left|y_{i}-y\right|}}{\kappa_{n}} \chi_{n}(x) \chi_{n}\left(x_{i}\right) \\
& \times \gamma\left(-i \frac{\mu}{\hbar^{2}}\right) \frac{e^{i \kappa_{m}\left|y-y_{i}\right|}}{\kappa_{m}} \chi_{m}(x) \chi_{m}\left(x_{i}\right),
\end{aligned}
$$

where $\chi_{n}(x)=\sqrt{2 / W} \sin (n \pi x / W)$ is the transverse wave function in mode $n \geqslant 1$, and $\mu$ is the electron effective mass. Taking into account multiple scattering by the impurity, the potential strength $\gamma$ is replaced with a $T$ matrix. When we take the imaginary part of the propagator to get the local density of states, we get spatially oscillating terms

$$
\propto \cos \left[\left(\kappa_{n}+\kappa_{m}\right)\left|y-y_{i}\right|\right]
$$

and

$$
\propto \sin \left[\left(\kappa_{n}+\kappa_{m}\right)\left|y-y_{i}\right|\right],
$$

since the $T$ matrix is a complex number due to multiple scattering. After Fourier transformation, we find peaks at $q_{y}=$ $\pm\left(\kappa_{n}+\kappa_{m}\right)$ and at $q_{x}$ equal to combinations of $n$ and $m$ times $\pi / W$. Thus, in a FT-STS picture of a quantum channel, there will be a discrete number of peaks that reflect the available 
modes. We also note that the Friedel oscillations (neglecting electron-electron interactions) will at low temperature oscillate without decay far from the impurity site.

To probe a 2DEG quantum channel with an STM in the way described here will be challenging since the channel is typically hidden deep down in a semiconducting heterostructure. Graphene, on the other hand, is $100 \%$ surface and directly accessible.

\section{FT-LDOS: RIBBON IN A 2DEG}

In this section we improve the above discussion to the general case of multiple scattering in a multimode 2DEG quantum channel of width $W$ with a single impurity scattering center at $\vec{r}_{i}$. The results of this section will be referenced in the following sections on graphene in order to highlight the distinguishing features of confined Dirac quasiparticles.

Consider the probability amplitude for an electron in the channel to propagate from one point $\vec{r}^{\prime}$ to another point $\vec{r}$. For free propagation in mode $n$, the amplitude is given by the free propagator (unperturbed Green's function), $g_{n}\left(\vec{r}, \vec{r}^{\prime} ; E\right)$. In the presence of the impurity an electron initially in mode $m$ may be scattered into mode $n$. The effect of such an extra process will modify the propagator by adding a second term,

$$
\tilde{G}_{n m}\left(\vec{r}, \vec{r}^{\prime} ; E\right)=g_{n}\left(\vec{r}, \vec{r}_{i} ; E\right) T\left(\vec{r}_{i} ; E\right) g_{m}\left(\vec{r}_{i}, \vec{r}^{\prime} ; E\right),
$$

so that the new Green's function will be

$$
G_{n m}\left(\vec{r}, \vec{r}^{\prime} ; E\right)=g_{n}\left(\vec{r}, \vec{r}^{\prime}, E\right) \delta_{n m}+\tilde{G}_{n m}\left(\vec{r}, \vec{r}^{\prime} ; E\right) .
$$

The factor $T\left(\vec{r}_{i} ; E\right)$ [see Eq. (A10)] includes multiple scattering by the impurity. The full probability amplitude for propagation from $\vec{r}^{\prime}$ to $\vec{r}$ is given by summing over all mode indices,

$$
G\left(\vec{r}, \vec{r}^{\prime} ; E\right)=\sum_{n m} G_{n m}\left(\vec{r}, \vec{r}^{\prime} ; E\right) .
$$

We may now proceed with the local density of states (LDOS). The correction to the LDOS by impurity scattering can be written as

$$
\begin{aligned}
\tilde{\rho}(\vec{r} ; E) & =-\frac{1}{\pi} \sum_{n m} \operatorname{Im} \tilde{G}_{n m}(\vec{r}, \vec{r} ; E) \\
& =-\frac{1}{\pi} \sum_{n m} \mathcal{K}_{n m}(E) \tilde{\rho}_{n m}^{x}(x ; E) \tilde{\rho}_{n m}^{y}(y ; E),
\end{aligned}
$$

where the expressions for the factors $\mathcal{K}_{n m}(E), \tilde{\rho}_{n m}^{x}(x)$, and $\tilde{\rho}_{n m}^{y}(y ; E)$ are given in Appendix A. The FT-LDOS can now be computed as

$$
\tilde{\mathcal{N}}(\vec{q} ; E)=-\frac{1}{\pi} \sum_{n m} \mathcal{K}_{n m}(E) \tilde{\mathcal{N}}_{n m}^{x}\left(q_{x}\right) \tilde{\mathcal{N}}_{n m}^{y}\left(q_{y} ; E\right),
$$

where

$$
\begin{aligned}
\tilde{\mathcal{N}}_{n m}^{x}\left(q_{x}\right) & =\sum_{l=-\infty}^{\infty} \delta\left(\frac{q_{x}}{\pi}-\frac{l}{W}\right) \int_{-W}^{W} \frac{d x}{2 W} e^{-i \frac{\pi}{W} l x} \tilde{\rho}_{n m}^{x}(x) \\
& =\sum_{l=-\infty}^{\infty} \delta\left(\frac{q_{x}}{\pi}-\frac{l}{W}\right) \tilde{\mathcal{N}}_{n m}^{x}(l)
\end{aligned}
$$

and

$$
\tilde{\mathcal{N}}_{n m}^{y}\left(q_{y} ; E\right)=\int_{-\infty}^{\infty} \frac{d y}{2 \pi} e^{-i q_{y} y} \tilde{\rho}_{n m}^{y}(y ; E) .
$$

The function $\tilde{\rho}_{n m}^{x}(x)$, originally defined on the interval $[0, W]$, is extended to $[-W, W]$ and assumed to be even with respect to the origin. Due to the finite width, $2 W$, of the integration interval, the spectral $x$ component is fixed to be integer multiples of $\pi / W$. This is a trick to be able to resolve the minimum change of transverse momenta, $\pi / W$, when scattering between two different modes.

It is important to realize that both propagating and evanescent modes play a role in this scattering problem. The longitudinal momentum is $\kappa_{n}=\sqrt{2 \mu E / \hbar^{2}-(n \pi / W)^{2}}$, where $\mu$ is the electron mass and $n \geqslant 1$ is the integer mode index. At the bottom of a subband, $\kappa_{n} \rightarrow 0$, and the evanescent mode extends far from the impurity and play an important role. On the other hand, for energies far from any subband bottom, the LDOS is only affected by the evanescent mode in a small region near the impurity. In the discussion of the FT-LDOS we can then safely neglect evanescent modes in the sums in Eq. (5). The evanescent modes are still taken into account in the scattering processes at the impurity through the $T$-matrix equation, where intermediate modes can be evanescent, while initial and final modes are propagating. In all of our numerical calculations, we include ten evanescent modes. Adding even more evanescent modes does not qualitatively change our results. As has been shown, a $\delta$-shaped impurity with a finite number of evanescent modes will model an $s$-like scatterer. ${ }^{29}$ We can now find the different components of the FT-LDOS to be

$$
\tilde{\mathcal{N}}_{n m}^{x}(l)=\frac{1}{2 W}\left(\delta_{l, n-m}+\delta_{-l, n-m}-\delta_{l, n+m}-\delta_{-l, n+m}\right)
$$

and

$\tilde{\mathcal{N}}_{n m}^{y}\left(q_{y}\right)=\frac{e^{-i y_{i} q_{y}}}{2 \pi}\left[S^{y}\left(\kappa_{n}+\kappa_{m}+q_{y}\right)+S^{y}\left(\kappa_{n}+\kappa_{m}-q_{y}\right)\right]$,

where

$$
S^{y}(a)=\lim _{\epsilon \rightarrow 0^{+}} \frac{\sigma_{p} \epsilon-a\left(1 / \gamma+\sigma_{e}\right)}{\epsilon^{2}+a^{2}}
$$

and where $\sigma_{p / e}$ are positive, $\vec{q}$-independent constants defined in Eq. (A11).

The factor $\mathcal{K}_{n m}(E)$ is given by

$$
\mathcal{K}_{n m}(E)=\frac{1}{\left(1 / \gamma+\sigma_{e}(E)\right)^{2}+\sigma_{p}^{2}(E)}\left(\frac{\mu}{\hbar^{2}}\right)^{2} \frac{\chi_{n}\left(x_{i}\right) \chi_{m}\left(x_{i}\right)}{\kappa_{n}(E) \kappa_{m}(E)}
$$

and depends on the scatterer strength $\gamma$ and the transversal wave functions $\chi_{n}(x)=\sqrt{2 / W} \sin (n \pi x / W)$.

Together, these components give rise to a number of selection rules that govern the modification of the FT-LDOS by impurity scattering. To illustrate, we select a narrow channel $W=50 a_{0}$, where $a_{0}$ defines the unit of length, and consider a low-energy $E=0.02 \tau$, where $\tau=\hbar^{2} /\left(2 \mu a_{0}^{2}\right)$ is the unit of energy, such that only a total of three propagating modes are open. The scattering FT-LDOS $|\tilde{\mathcal{N}}(\vec{q} ; E)|$ for the case of the impurity in the middle of the ribbon $\left(x_{i}=W / 2, y_{i}=0\right)$ is 

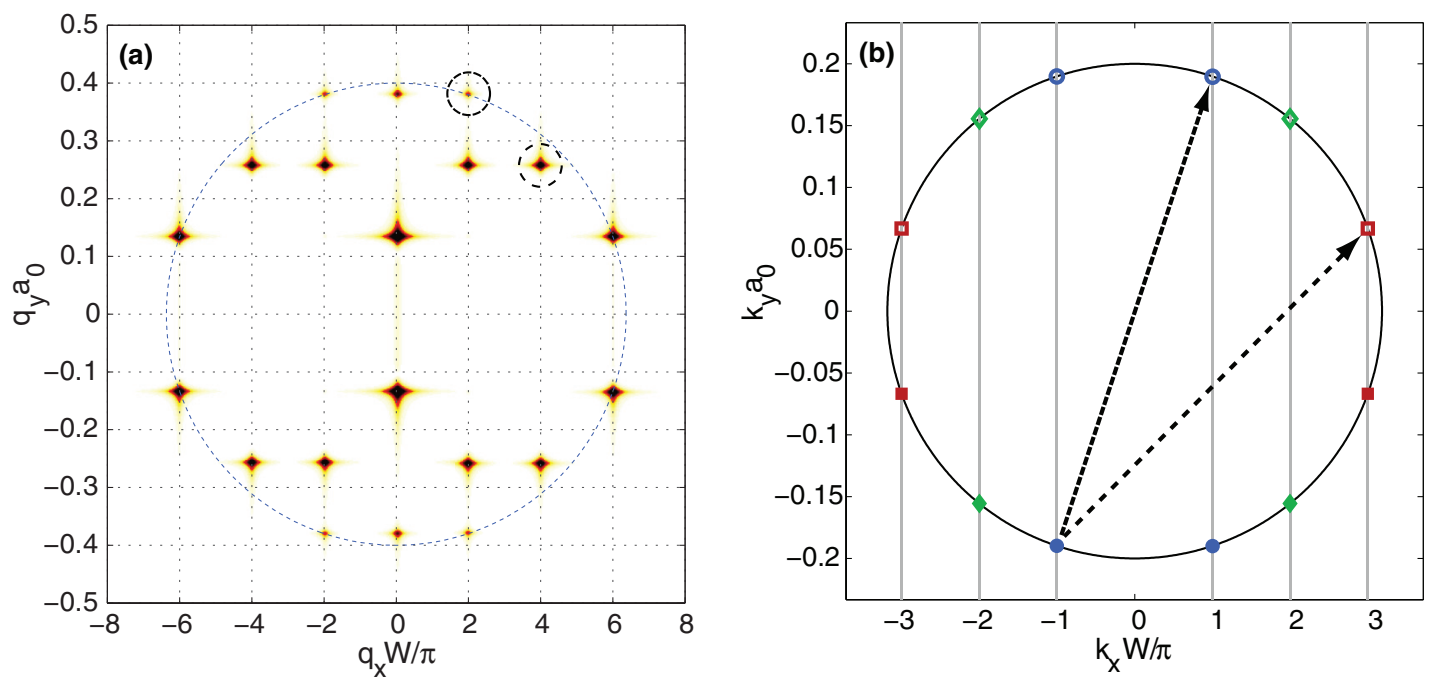

FIG. 2. (Color online) (a) FT-LDOS of a 2DEG ribbon of width $W=50 a_{0}$ at energy $E=0.02 \tau$, with three open (propagating) modes. (b) Energy contour of the 2DEG dispersion. The symbols indicate the allowed momentum values between which scattering can potentially take place (circles, diamonds, and squares correspond to $n=1, n=2$, and $n=3$, respectively). The two arrows in figure (b) illustrates two possible scattering processes that gives rise to the two encircled dots in figure (a). The impurity is placed at $x_{i}=W / 2$ and the $n=2$ subband is not scattered by the impurity because the impurity has been located at a node of the corresponding transverse wave function.

displayed in Fig. 2(a). Since the scattering is elastic, energy conservation requires that the transverse and longitudinal momenta, both before and after scattering, satisfy the relation $2 \mu E / \hbar^{2}=k_{x}^{2}+\kappa_{n}^{2}(E)$, which is the circle shown in Fig. 2(b). In the channel, the transverse momentum is quantized, $k_{x} \rightarrow$ $k_{n}=n \pi / W$, and the only allowed momentum values between which the electrons can scatter are indicated by dots and squares on this circle. The FT-LDOS is therefore nonzero only at a few, finite number of $\vec{q}$ points. All of these points lie inside the dotted circle of radius $2 \sqrt{2 \mu E / \hbar^{2}}$ shown in Fig. 2(a).

The impurity scattering strength $\gamma$ can be either positive or negative, corresponding to a repulsive or attractive impurity potential. For the FT-LDOS, the sign of $\gamma$ does not play a major role. In Fig. 2 and below, we choose a repulsive $\gamma=10 \tau$.

The factor $\mathcal{K}_{n m}(E)$ will be nonzero only if the transverse wave functions of mode $n$ and $m$ have a finite overlap at the position of the impurity. Since we have positioned the impurity at $x_{i}=W / 2, \mathcal{K}_{n m}(E)$ will in this example be nonzero only if $n$ and $m$ are both odd integers since all the wave functions with even indices will have a node at $x=x_{i}$. Thus, modes with even number $n$ are not scattered by the impurity in this example.

To understand the exact locations of the $\vec{q}$ points, we start by looking at the case $q_{x}=0$ (i.e., $l=0$ ). Since all mode indices have to be odd, the term $\tilde{\mathcal{N}}^{x}(l=0)$ will be nonzero only when $n=m$, i.e., when $(n=1, m=1)$ or when $(n=$ $3, m=3)$. This tells us that the points along $q_{x}=0$ are all due to intraband scattering. The factor $\tilde{\mathcal{N}}^{y}\left(q_{y} ; E\right)$ peaks when $q_{y}= \pm 2\left|\kappa_{1}\right|$ or when $q_{y}= \pm 2\left|\kappa_{3}\right|$. These are the four points we see along the line $l=0$.

When $q_{x}=\pi / W(l=1)$, at least one of the indices $n$ and $m$ will be even, and the factor $\mathcal{K}_{n m}(E)$ is zero. This is why we see no bright points along this line. This also happens for $l=3$ and $l=5$.

Along the line $q_{x}=2 \pi / W$, we have that $\tilde{\mathcal{N}}^{x}(l=2)$ is nonzero only when $(n=1, m=1),(n=1, m=3)$ or when $(n=3, m=1)$. The factor $\tilde{\mathcal{N}}^{y}\left(q_{y} ; E\right)$ peaks at $q_{y}= \pm 2\left|\kappa_{1}\right|$ or when $q_{y}= \pm\left|\kappa_{1}+\kappa_{3}\right|$, and we see that we have spots at these locations along $l=2$ in the figure.

At $l=4$ we must have $(n=3, m=1)$ or $(n=1, m=3)$, which tells us that $q_{y}= \pm\left|\kappa_{3}+\kappa_{1}\right|$. At $l=6$, we must have $(n=3, m=3)$ and $q_{y}= \pm 2\left|\kappa_{3}\right|$. A similar argument can be made for $l<0$, and we can therefore say exactly which scattering processes contribute to each dark spot in Fig. 2(a).

If the impurity is not located exactly at the middle of the ribbon, the even subbands will also be part of the scattering process. This is illustrated in Fig. 3, where we have numbered the subband transitions corresponding to each bright point.

In Fig. 4, we show the result for a wider ribbon calculated both analytically and by doing a recursive tight-binding

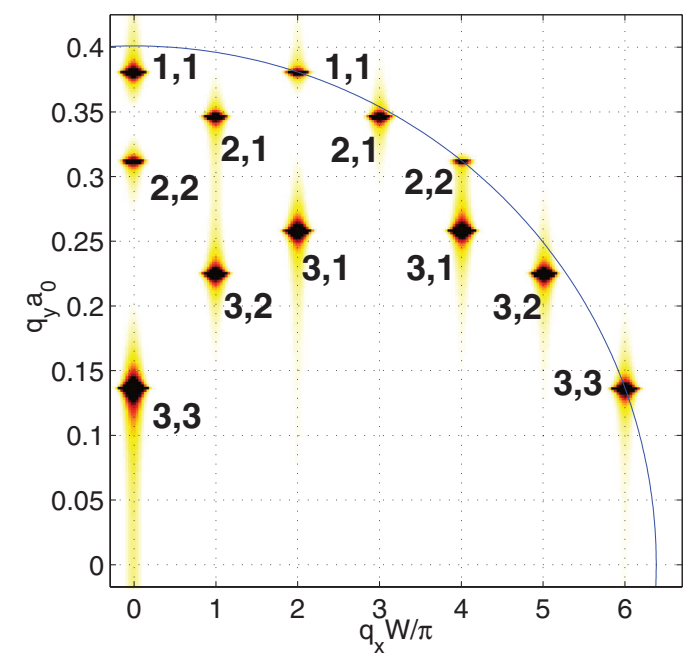

FIG. 3. (Color online) The first quadrant of the FT-LDOS of the same ribbon as in Fig. 2, but with the impurity placed at $x_{i}=2 \mathrm{~W} / 7$. The numbers indicate the modes that gives rise to the different points. 

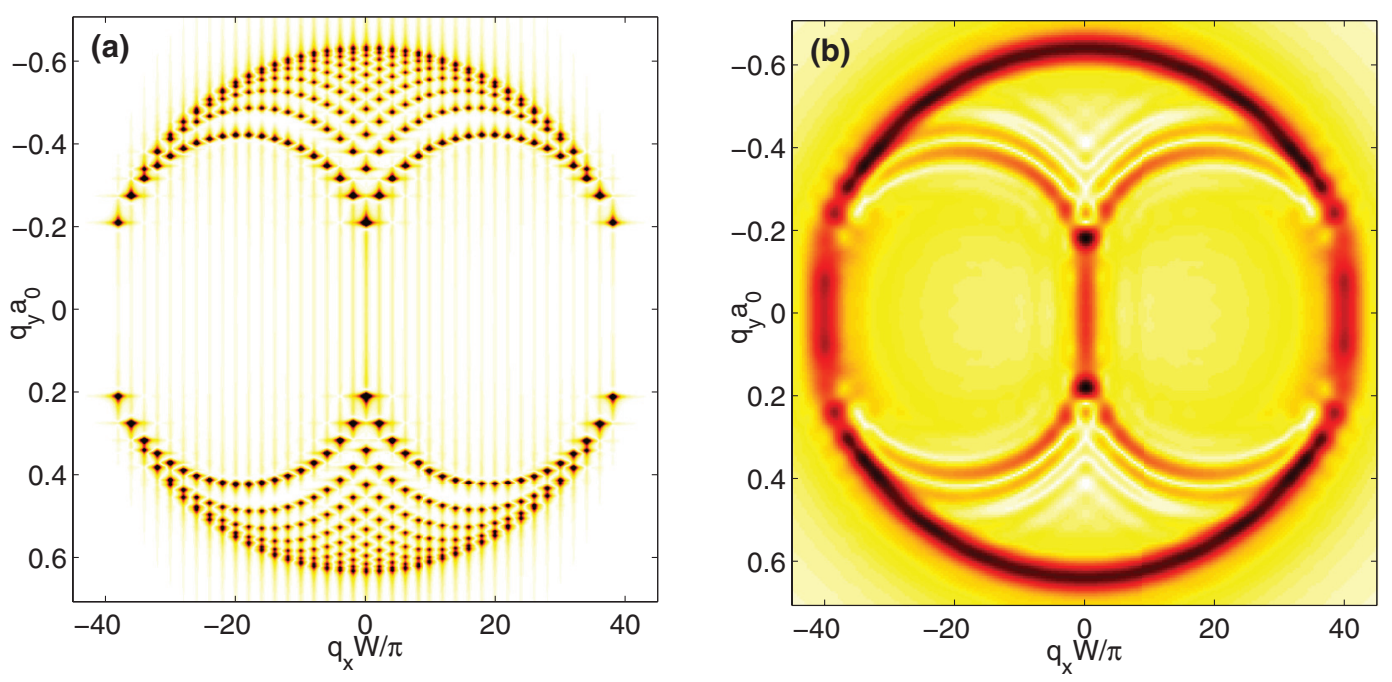

FIG. 4. (Color online) (a) Analytical FT-LDOS of a 2DEG ribbon of width $W=200 a_{0}$ and energy $E=0.05 \tau$. (b) FT-LDOS taken from a numerical tight-binding simulation of a ribbon of width $W=200 a_{0}$ and energy $E_{T B}=-3.9 \tau$. The energies are adjusted such that each ribbon has a total of 20 propagating modes open.

simulation. In the analytic model (which has a parabolic dispersion), setting the energy to $E=0.05|\tau|$ gives us a total of 20 open propagating modes. In the tight-binding model (see Sec. V), whose first subband is located around $E=-4|\tau|$ (if we set the on-site energy to zero everywhere), we must instead choose the energy to be $E_{T B}=-3.9|\tau|$ in order to have the same number (20) of open modes. We see that the main features of our analytical calculation and the numerical simulation coincide.

\section{FT-LDOS: RIBBONS OF GRAPHENE}

The procedure of calculating the effect of a single impurity (located on the A sublattice) on the LDOS in a graphene

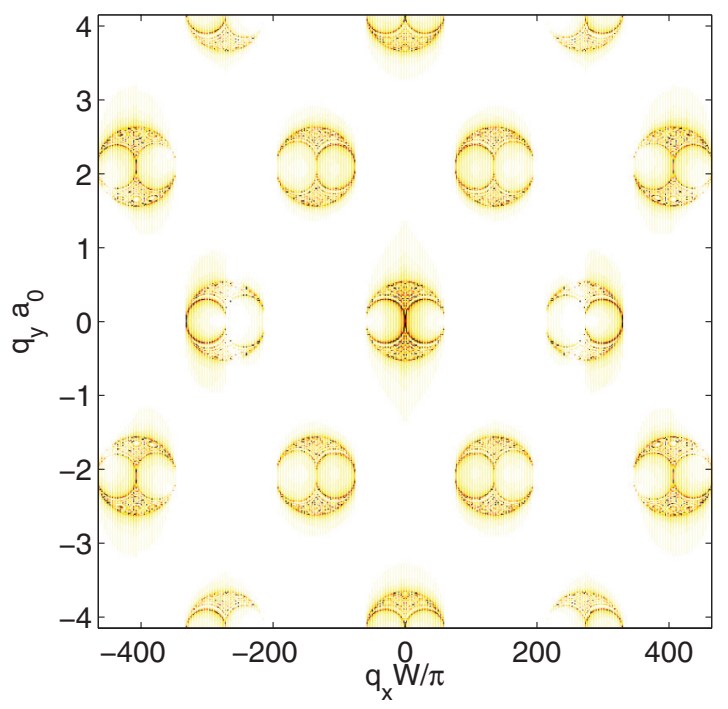

FIG. 5. (Color online) Analytic FT-LDOS, $\tilde{\mathcal{M}}(\vec{q} ; E)$, of an AGNR having a width $W=203 a(\approx 50 \mathrm{~nm}), E=0.4 \hbar v_{f} / a_{0}, \gamma=$ $10 \hbar v_{f} / a_{0}$ and 60 propagating channels. armchair nanoribbon (AGNR) follows closely that used for the $2 \mathrm{DEG}$ case. Due to the bipartite structure of the graphene honeycomb lattice, the propagator $\tilde{\mathbf{G}}\left(\vec{r}, \vec{r}^{\prime} ; E\right)$ is a $2 \times 2$ matrix in sublattice space, denoted by A and B. We therefore start by finding the impurity contribution to the LDOS on each sublattice. The resulting expressions for the A- and B-sublattice LDOS can be written as

$$
\begin{aligned}
\tilde{\rho}^{A / B}(\vec{r} ; E) & =-\frac{1}{\pi} \sum_{d c} \sum_{n m} \operatorname{Im} \tilde{G}_{d n c m}^{A A / B B}(\vec{r}, \vec{r} ; E) \\
& =-\frac{1}{\pi} \sum_{d c} \sum_{n m} \mathcal{K}_{d n c m}^{A / B}(E) \tilde{\rho}_{n m}^{x}(x ; E) \tilde{\rho}_{d n c m}^{(A / B) y}(y ; E),
\end{aligned}
$$

where $\tilde{G}_{d n c m}^{A A / B B}(\vec{r}, \vec{r} ; E)$ are the two diagonal components of the propagator matrix $\tilde{\mathbf{G}}_{d n c m}(\vec{r}, \vec{r} ; E)$. The summation over the variables $c$ and $d$ are added to account for scattering between different sets of nonequivalent Dirac cone pairs $\vec{K}_{c}^{ \pm}$ and $\vec{K}_{d}^{ \pm}$. A further elaboration on this is found in Appendix B, together with derivations of the expressions for $\tilde{\rho}_{n m}^{x}(x ; E)$ and

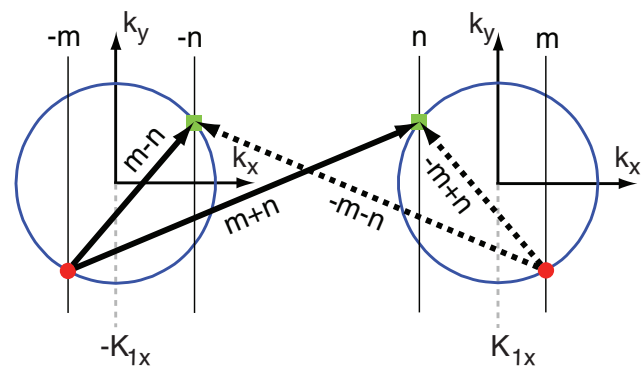

FIG. 6. (Color online) Schematic picture of one possible scattering process in AGNRs. Here, the electron (initially in mode $m$, represented by the red dots), is scattered into mode $n$ (green squares). The FT-LDOS will be finite at the $\vec{q}$ values illustrated by the solid $\left[q_{x}=(m \pm n) \pi / W\right]$ and dotted $\left[q_{x}=-(m \pm n) \pi / W\right]$ arrows. 

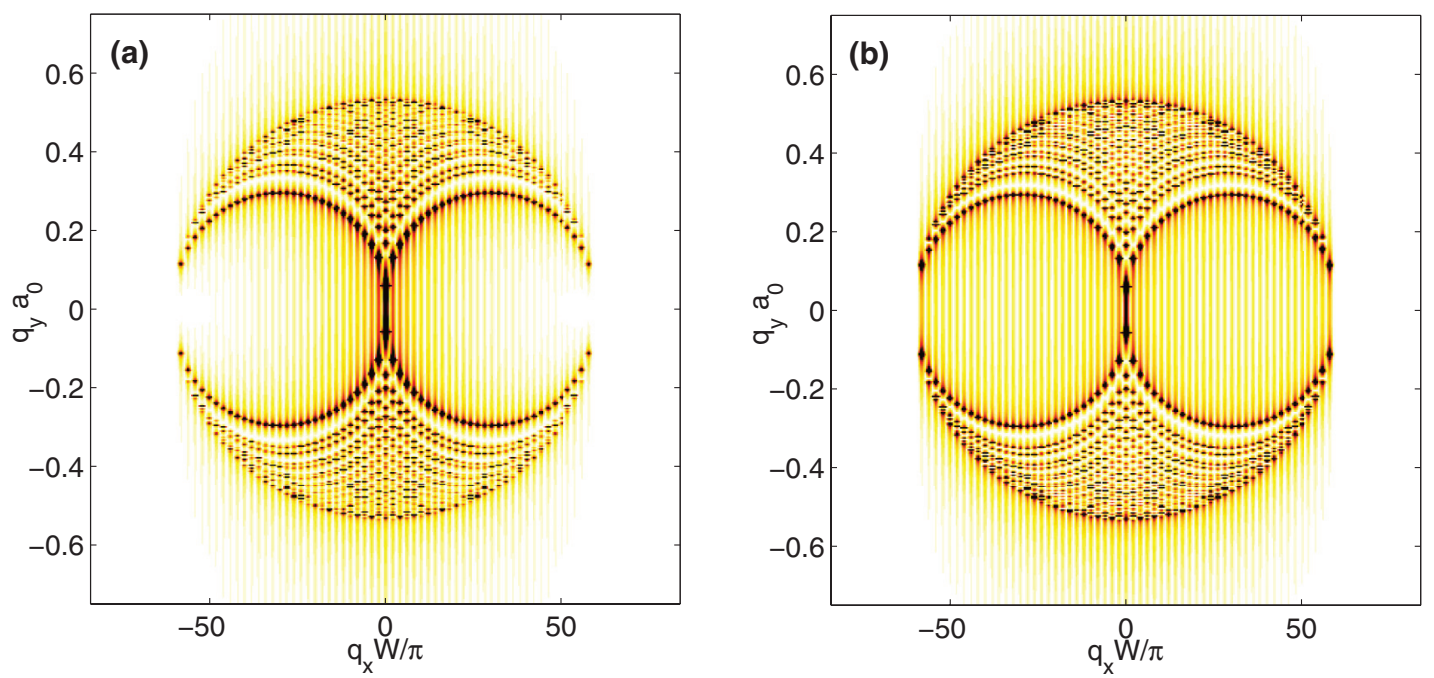

FIG. 7. (Color online) Magnifications of the central circular feature of Fig. 5 showing (a) the combined A- and B-lattice contributions and (b) the A lattice alone. When adding the two lattices together, the outer circle is attenuated by destructive interference between the two lattice contributions, $\tilde{\mathcal{M}}^{(A / B) y}\left(q_{y} ; E\right)$, to the total FT-LDOS.

$\tilde{\rho}_{d n c m}^{(A / B) y}(y ; E)$. As discussed in Sec. III, we only need to sum over propagating incoming and final transverse modes, labeled by $m$ and $n$.

We compute the FT-LDOS on each sublattice as

$$
\tilde{\mathcal{M}}^{A / B}(\vec{q} ; E)=-\frac{1}{\pi} \sum_{n m} \tilde{\mathcal{M}}_{n m}^{x}\left(q_{x} ; E\right) \tilde{\mathcal{M}}_{n m}^{(A / B) y}\left(q_{y} ; E\right) .
$$

The total FT-LDOS is found as a superposition of the two sublattices

$$
\tilde{\mathcal{M}}(\vec{q} ; E)=\tilde{\mathcal{M}}^{A}(\vec{q} ; E)+e^{-i a_{0} q_{y}} \tilde{\mathcal{M}}^{B}(\vec{q} ; E),
$$

where the extra phase-shift is introduced since the two sublattices are spatially separated by the carbon-carbon distance $a_{0}$ in the $y$ direction.

Since the transverse wave functions, $\chi_{n}(x)=$ $\sqrt{1 / W} \sin (n \pi / W x)$, in our AGNR differ from those of the 2DEG only by a factor of $1 / \sqrt{2}$, we have that $\tilde{\mathcal{M}}_{n m}^{x}\left(q_{x} ; E\right)=\tilde{\mathcal{N}}_{n m}^{x}\left(q_{x} ; E\right) / 2$, as defined in Eqs. (6) and (8).

The longitudinal FT-LDOS expressions for each sublattice are given by

$$
\begin{aligned}
\tilde{\mathcal{M}}_{n m}^{(A / B) y}\left(q_{y} ; E\right) \\
=\frac{e^{-i q_{y} y_{i}}}{2 \pi} \sum_{c=1}^{3} \mathcal{K}_{c n c m}^{A / B}(E)\left[S^{(A / B) y}\left(\Delta_{c n c m}(E)-q_{y}\right)^{*}\right. \\
\left.\quad+S^{(A / B) y}\left(\Delta_{c n c m}(E)+q_{y}\right)\right]+\frac{e^{-i q_{y} y_{i}}}{2 \pi} \sum_{d=1}^{2} \sum_{c=d+1}^{3} \mathcal{K}_{d n c m}^{A / B}(E) \\
\quad \times\left[S^{(A / B) y}\left(\Delta_{c n d m}(E)-q_{y}\right)^{*}+S^{(A / B) y}\left(\Delta_{d n c m}(E)-q_{y}\right)^{*}\right. \\
\left.\quad+S^{(A / B) y}\left(\Delta_{c n d m}(E)+q_{y}\right)+S^{(A / B) y}\left(\Delta_{d n c m}(E)+q_{y}\right)\right],
\end{aligned}
$$

where $\quad \Delta_{d n c m}(E)=\operatorname{sgn}(E)\left[\kappa_{d n}(E)+\kappa_{d m}(E)\right]+K_{d}^{y}-K_{c}^{y}$. The two $\vec{q}$-independent constants are found to be

$$
\begin{aligned}
\mathcal{K}_{d n c m}^{A}(E)= & \frac{1}{\left[1 / \gamma+\sigma_{e}(E)\right]^{2}+\sigma_{p}^{2}(E)}\left(\frac{|E|}{\left(\hbar v_{f}\right)^{2}}\right)^{2} \\
& \times \frac{\chi_{n}\left(x_{i}\right) \chi_{m}\left(x_{i}\right)}{\kappa_{d n}(E) \kappa_{c m}(E)}
\end{aligned}
$$

and $\mathcal{K}_{d n c m}^{B}(E)=-\left(\hbar v_{f} /|E|\right)^{2} \mathcal{K}_{d n c m}^{A}(E)$. The transverse and longitudinal momenta are now cone set dependent and change to $k_{d n}=n \pi / W-K_{d x}$ and $\kappa_{d n}(E)=\sqrt{\left[E /\left(\hbar v_{f}\right)\right]^{2}-k_{d n}^{2}}$, respectively. Here, $S^{A y}(a)=S^{y}(a)$ is the same function as used in the 2DEG case and defined in Eq. (10), and $S^{B y}(a)=$ $f_{d n c m}(E) S^{y}(a)$, where $f_{d n c m}(E)=\left[-k_{n} k_{m}+\kappa_{n}(E) \kappa_{m}(E)\right]+$ $i \operatorname{sgn}(E)\left[k_{n} \kappa_{m}(E)+k_{m} \kappa_{n}(E)\right]$. The $S$-terms for the AGNR A lattice have the exact same form as the corresponding terms in the 2DEG, while the B lattice terms are scaled by a complex mode dependent prefactor. This is a consequence of our choice of impurity potential: an impurity fully localized on one A atom; see Eq. (B11).

In Fig. 5, we plot $|\tilde{\mathcal{M}}(\vec{q} ; E)|$ for a semiconducting AGNR of width $W=203 a\left(\approx 50 \mathrm{~nm}, a=\sqrt{3} a_{0}\right)$, where $E=$ $0.4 \hbar v_{f} / a_{0}$ such that 60 channels are propagating. Note that the natural energy scale for Dirac electrons are $\hbar v_{f} / a_{0}$. The positions, outer shapes, and sizes of the circular features [of radius $\left.2 E /\left(\hbar v_{f}\right)\right]$ are the same as those found when studying a sharp impurity in bulk graphene and are due to the graphene band structure. In addition to the bulk graphene features, we also see an added rich inner structure due to transverse confinement in the nanoribbon. Each peak corresponds to scattering processes that change the transverse momentum by integer multiples of $\pi / W$, and change the longitudinal momentum such that the arguments of at least one of the many $S^{y}$ terms in Eq. (15) vanishes.

A schematic illustration of one such scattering process is shown in Fig. 6. An electron, initially in cone pair $c=1$ and mode $m$, is described by a plain wave with momentum $-\kappa_{1 m}$ 

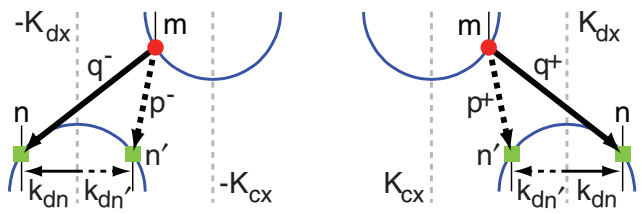

FIG. 8. (Color online) Schematic picture of scattering processes conserving mirror symmetry with respect to $q_{x}=0$ (solid arrows) and with respect to $q_{x}= \pm K_{d x}$ (solid + dotted arrows).

in the longitudinal direction and a superposition of two plain waves with momenta $\pm k_{1 m}$ in the transverse direction (see the lower red dots). After scattering (within the same cone pair) to mode $n$, the momenta are changed to $\kappa_{1 n}$ and $\pm k_{1 n}$ in the longitudinal and transverse directions, respectively (see the upper green squares). A Fourier transform of the LDOS is proportional to a product of the electron wave function before and after the scattering event, where each wave function is a linear combination of two transverse parts. The FT-LDOS will therefore be finite at the $\vec{q}$ values corresponding to the four arrows shown in the figure. Here, $q_{y}=\left|\kappa_{1 n}+\kappa_{1 m}\right|$, and $q_{x}=$ $(m \pm n) \pi / W$ (solid arrows) or $q_{x}=-(m \pm n) \pi / W$ (dotted arrows). When scattering to a different cone pair $d \neq c$, we instead have $q_{y}=\left|\Delta_{d n 1 m}\right|$, see Eq. (15).

When we zoom in on the circular feature in the middle [shown in Fig. 7(a)] we see that the outer ring of nonvanishing $\vec{q}$ points in $|\tilde{\mathcal{M}}(\vec{q} ; E)|$ appears to be attenuated compared with what is seen on, e.g., the A lattice alone $\left[\left|\tilde{\mathcal{M}}^{A}(\vec{q} ; E)\right|\right.$ shown in Fig. 7(b)]. This is due to destructive interference when adding the A- and B-lattice FT-LDOS contributions together, as done in Eq. (14). The $\vec{q}$ points on the outer circle come from scattering processes which maximize the change in momenta while still scattering within the same cone pair, i.e., where $d=c$ and $k_{c m} \rightarrow-k_{d n}$ and vice versa. In this case, we have that $f_{d n c m}=\left[E /\left(\hbar v_{f}\right)\right]^{2}$, which tells us that $\tilde{\mathcal{M}}_{n m}^{B y}\left(q_{y} ; E\right)=$ $-\tilde{\mathcal{M}}^{A y}\left(q_{y} ; E\right)$ so that when the phase factor $e^{-i q_{y} a}$ in Eq. (14) is close to unity, the contributions from the A and B lattices will cancel each other out. Similar cancellations may be seen

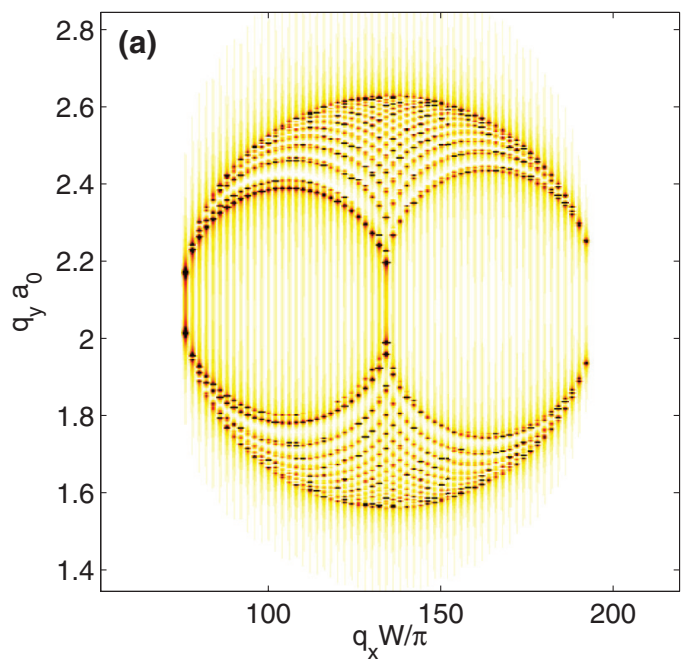

in Fig 5, e.g., in the circular features to the right and the left of the central one. For other processes and $\vec{q}$ values, the interference between the two lattice contributions may not play an important role, or we might have constructive interference instead.

The FT-LDOS is left-right mirror symmetric around the line $q_{x}=0$; see Fig. 5. This symmetry appears because for every process adding a component $\vec{q}^{+}$in the FT-LDOS, there is another process adding a component $\vec{q}^{-}$, where $q_{x}^{-}=-q_{x}^{+}$, see the solid arrows in Fig. 8. After summation of all such processes, the FT-LDOS acquires the left-right symmetry.

As a consequence, the feature centered around $\vec{q}=0$ is always mirror symmetric by the above argument. On the other hand, there is not necessarily a mirror symmetry within the other circular features (i.e., mirror symmetry with respect to the individual cone centers). For metallic AGNRs, the transverse modes are constructed from wave vectors symmetrically positioned with respect to the cone center (plus the metallic mode at the cone center). See, for instance, the two wave vectors $k_{d n}$ and $k_{d n^{\prime}}=-k_{d n}$ in Fig. 8. For semiconducting AGNRs, the wave vectors are not symmetrically positioned with respect to the cone center, i.e., $k_{d n^{\prime}} \neq-k_{d n}$ for any $n^{\prime}$. Therefore, the inner structure of the circular features centered at finite $\vec{q}$ are symmetric for metallic AGNRs and asymmetric for semiconducting AGNRs. This is illustrated in Fig. 9 for the semiconducting and metallic cases in panels (a) and (b), respectively. We conclude that by looking at what symmetries there are in the FT-LDOS, one can extract information about whether an AGNR is metallic or not.

\section{NUMERICAL SIMULATIONS OF THE TIGHT-BINDING MODEL}

For our numerical simulations, we use a tight-binding model described by the Hamiltonian

$$
H=\sum_{i=1}^{N} \epsilon_{i} c_{i}^{\dagger} c_{i}+\sum_{i \neq j}^{N} t_{i j} c_{i}^{\dagger} c_{j},
$$

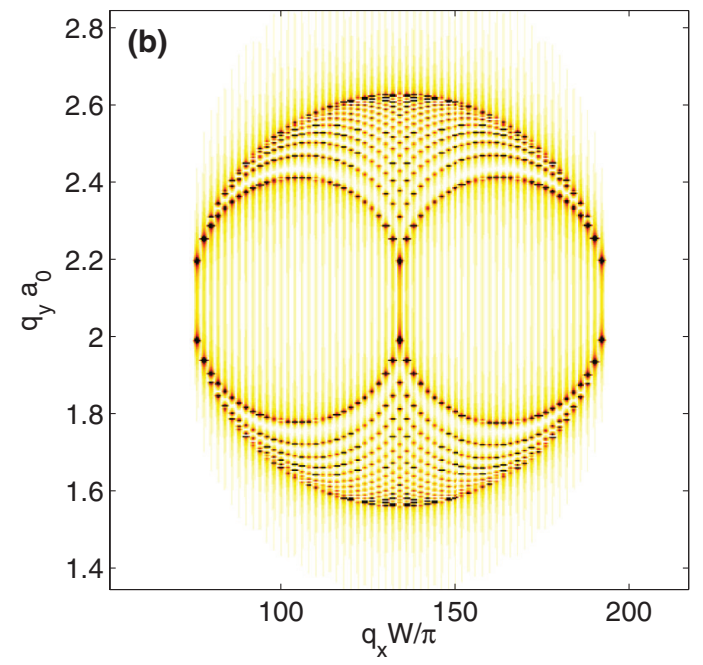

FIG. 9. (Color online) Zoom-ins of the north-eastern circular feature in Fig. 5. In (a), the AGNR is semiconducting and the left- and right-hand side is not mirror-symmetric. In (b), the ribbon is made metallic by removing four rows of carbon atoms, which restores the left-right symmetry again. 
where $c_{i}^{\dagger}$ and $c_{i}$ are creation and destruction operators for site $i$. The onsite energy of site $i$ is denoted $\epsilon_{i}$, and the hopping amplitude between sites $j$ and $i$ is denoted $t_{i j}$. The number of atoms in the system is denoted $N$. We assume that $t_{i j}$ is always zero except when the sites $i$ and $j$ are nearest neighbors where we set $t_{i j}=-\tau$. We introduce a $\delta$-like impurity in the tight-binding model by putting the on-site energy $\epsilon_{i}$ equal to $\gamma$ at the site of the impurity.

The retarded Green's function matrix is defined as

$$
\mathbf{G}(E)=[(E+i \eta) \mathbf{1}-\mathbf{H}]^{-1},
$$

where $\eta$ is a small positive number. Even though the Hamiltonian is sparse, when written down as a matrix in site index space, direct inversion is not a viable alternative when the number of atoms $N$ grows large. Instead of direct matrix inversion, we use our own implementation of a recent algorithm ${ }^{30}$ in which the system atoms are added one by one, in a recursive manner. This allows us to save both memory and time, and once we have found all the retarded propagators between the system leads and atom $i$ we can calculate the lesser Green's function, defined as

$G_{i i}^{<}(E)=\sum_{l} f_{l}(E) \sum_{\alpha_{l} \beta_{l}} G_{i \alpha_{l}}(E)\left[\Sigma_{l}^{\dagger}(E)-\Sigma_{l}(E)\right]_{\alpha_{l} \beta_{l}} G_{\beta_{l} i}^{\dagger}(E)$,

where $l$ is the lead number $(l=1,2$ in the case of a simple ribbon), and $\alpha_{l}$ and $\beta_{l}$ are indices running over all atoms belonging to the surface of lead $l$. Here, $f_{l}(E)$ and $\Sigma_{l}(E)$ are the distribution function and the self-energy of lead $l$, respectively.

The LDOS on atom $i$ is found from

$$
\rho_{i}(E)=-\frac{1}{\pi} \operatorname{Im} G_{i i}^{<}(E),
$$

and the FT-LDOS is given by doing a discrete Fourier transform over all system atoms,

$$
\mathcal{N}(\vec{q} ; E)=\frac{1}{N} \sum_{i=1}^{N} e^{-i \vec{r}_{i} \cdot \vec{q}} \rho_{i}(E),
$$

where $\vec{r}_{i}$ is the real space coordinate vector of atom $i$.

In Fig. 10(a), the result of such a tight-binding simulation is shown for a ribbon and setup matching the one used in Fig. 5, with a $\delta$-like impurity placed in the middle $(W=$ $203 a \approx 50 \mathrm{~nm}, 60$ propagating channels and $x_{i}=W / 2$ ). Upon inspection, we notice that the general features are similar compared with our analytical results. Some points, such as the outline of the central circle, are attenuated. The tight-binding ribbon does, however, show clear signs of trigonal warping due to the dispersion not being perfectly linear. In Fig. 10(b), we have moved the impurity to the edge of the ribbon and we notice that the resulting FT-LDOS image is not very different from the one with the impurity in the middle of the ribbon. In Fig. 10(c), we have again put the impurity in the middle of the ribbon, but instead made it Gaussian shaped (with a decay length that exceeds the spacing between two adjacent carbon atoms). This (long-range) impurity can be seen to suppress scattering and attenuate the FT-LDOS features, although not completely diminish them. For bulk graphene, it is well known that a long-range impurity cannot scatter between valleys.
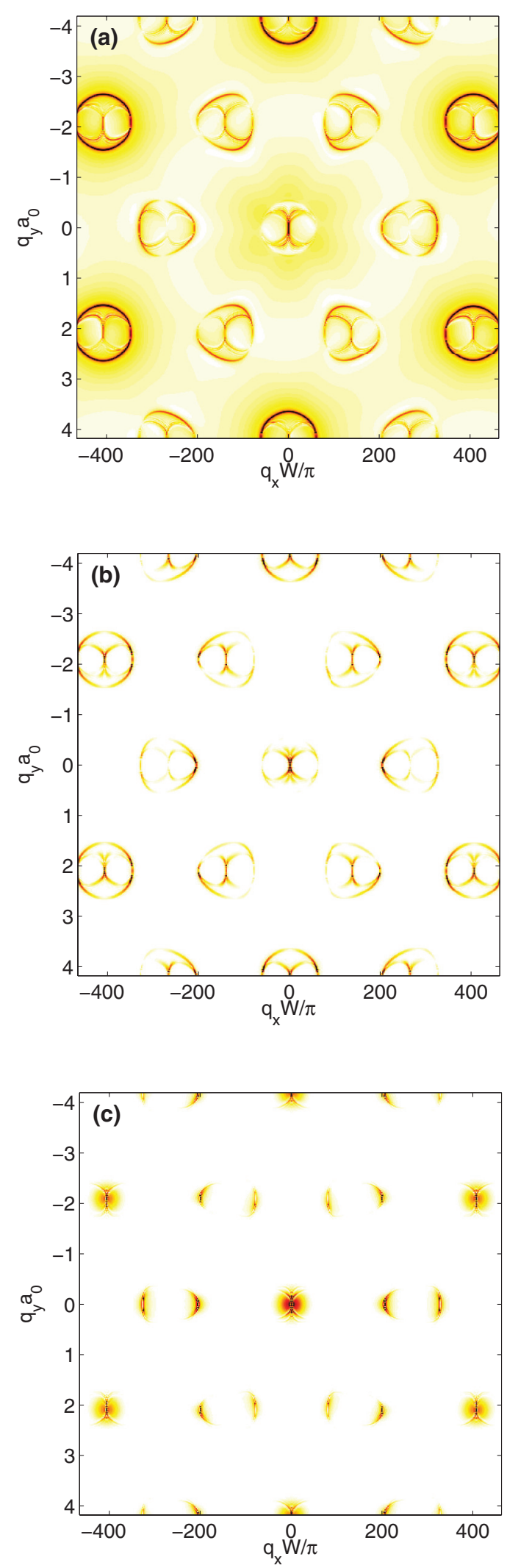

FIG. 10. (Color online) Numerical tight-binding FT-LDOS of three AGNRs' $(N=810$ atoms in the unit cell) with different impurity configurations. (a) Single impurity, (b) edge impurity, (c) smooth (Gaussian long-range) impurity, where $W=$ $203 a(\approx 50 \mathrm{~nm}), \gamma=10 \tau, E_{T B}=0.4 \tau$, and 60 propagating channels. 

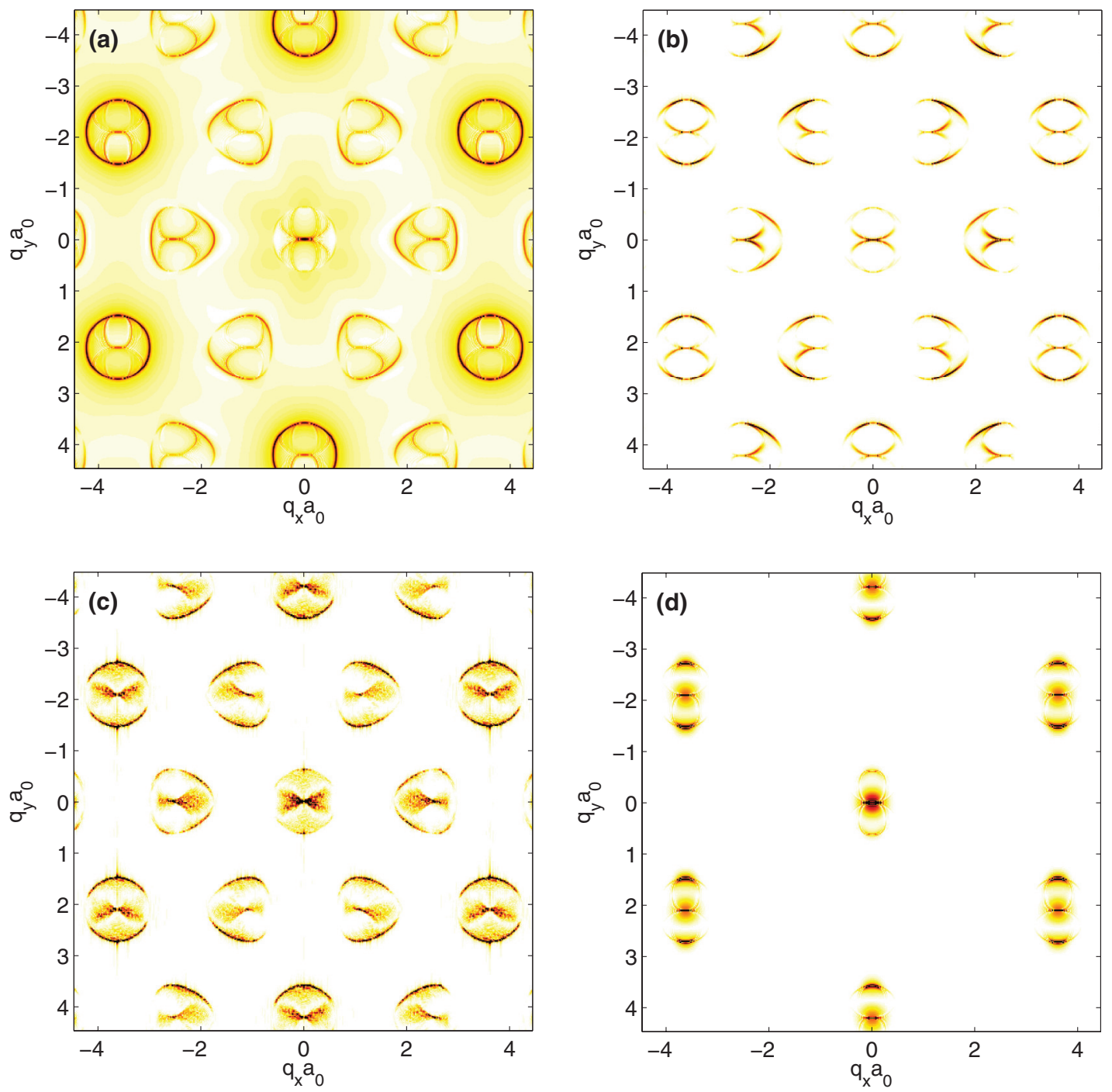

FIG. 11. (Color online) Numerical tight-binding FT-LDOS of four ZGNRs ( $N=468$ atoms in the unit cell) with different impurity configurations. (a) Single impurity, (b) edge impurity, (c) rough edges, (d) smooth impurity (Gaussian long-range), where $W=352 a_{0}$ $(\approx 50 \mathrm{~nm}), E_{T B}=0.45 \tau, \gamma=10 \tau$, and 35 propagating channels.

In the FT-LDOS, the features centered at $\vec{q}=\vec{K}_{d}^{p m}$ are then absent. This is not the case here, since the armchair nanoribbon has only one cone in its band structure. ${ }^{31}$

In Fig. 11, we present results for the FT-LDOS of zigzag graphene nanoribbons (ZGNRs). In this simulation the ribbon has $N=468$ atoms in its unit cell $\left(W=352 a_{0} \approx 50 \mathrm{~nm}\right)$, $\gamma=10 \tau$, and $E=0.45 \tau$. This gives 35 propagating modes. In Fig. 11(a), the impurity is located in the middle of the ribbon and we see a pattern very similar to that of the same impurity configuration in an armchair ribbon, but with all features rotated $90^{\circ}$ due to the different ribbon alignment (for ZGNRs, $k_{y}$ is quantized instead). The result of a single impurity on the edge is shown in Fig. 11(b), and in Fig. 11(c) we show the spectra for a ribbon also having rough edges. In the last figure, Fig. 11(d), we have again used a Gaussian-shaped (long-range) impurity located in the center of the ribbon, and we here see clearly that intervalley scattering is now fully supressed. ${ }^{32}$ Indeed, since the ZGNR has two cones in its band structure, this case is similar to bulk graphene.

\section{SUMMARY}

In summary, we have presented results for the FT-LDOS of graphene nanoribbons with local scattering centers. The interplay between size quantization and scattering leads to characteristic peaks that can be related to the transverse modes of the nanoribbon. The main features include ringlike structures, analogous to the case of an infinite 2D graphene sheet with a single scattering center. Inside the ringlike structure, new peaks appear that are related to inter- and intraband scattering in the ribbon. We have presented analytic results for the electron propagator in armchair nanoribbons in the Dirac approximation, including a single scattering center within a $T$-matrix formulation. We have also extended the investigation with numerics obtained with an atomistic recursive Green's function approach. The spectral signatures of the atomistic approach include the lifting of degeneracies of transverse modes in the Dirac approximation, as well as effects of trigonal warping. The impurity induced oscillations 
in the LDOS are not decaying at large distance in few-mode nanoribbons.

Future extensions of this work could involve consideration of bilayer graphene ribbons, as in Ref. 33, or more realistic modeling of the impurity potential, as considered, for instance, in Refs. 34 and 35.

\section{ACKNOWLEDGMENTS}

This work has been supported by SSF, the Swedish Foundation for Strategic Research, and the EU through the FP7 Project ConceptGraphene.

\section{APPENDIX A: NANORIBBON IN A 2DEG}

\section{Unperturbed Green's function}

For a 2DEG confined in the $x$ direction, creating a ribbon of width $W$, the wave functions can be written as

$$
\phi_{n}(\vec{r})=e^{i k_{y} y} \chi_{n}(x),
$$

where $\vec{r}=(x, y)$ and $n$ is the mode number associated with the transverse eigenfunctions (assuming infinitely high confining walls at $x=0$ and $x=W$ ) given by

$$
\chi_{n}(x)=\sqrt{\frac{2}{W}} \sin \left(k_{n} x\right),
$$

with the corresponding eigenenergies

$$
\epsilon_{n}\left(k_{y}\right)=\frac{\hbar^{2}}{2 \mu}\left(k_{n}^{2}+k_{y}^{2}\right) .
$$

Here, $k_{n}=n \pi / W$ is the transverse momentum, $k_{y}$ the longitudinal momentum, and $\mu$ the electron mass. Using these wave functions we may construct the free propagator, or Green's function, of an electron (having energy $E^{+}=E+i \eta$, in the limit $\eta \rightarrow 0^{+}$) between the points $\vec{r}^{\prime}$ and $\vec{r}$ as

$$
\begin{aligned}
g\left(\vec{r}, \vec{r}^{\prime} ; E\right) & =\sum_{n} \int_{-\infty}^{\infty} \frac{d k_{y}}{2 \pi} \frac{\phi_{n}(\vec{r}) \phi_{n}^{*}\left(\vec{r}^{\prime}\right)}{E^{+}-\epsilon_{n}\left(k_{y}\right)} \\
& =\sum_{n} \underbrace{\chi_{n}(x) \chi_{n}\left(x^{\prime}\right) \Gamma_{n}\left(y, y^{\prime} ; E\right)}_{=g_{n}\left(\vec{r}, \vec{r}^{\prime} ; E\right)},
\end{aligned}
$$

where

$$
\Gamma_{n}\left(y, y^{\prime} ; E\right)=\int_{-\infty}^{\infty} \frac{d k_{y}}{2 \pi} \frac{e^{i k_{y}\left(y-y^{\prime}\right)}}{E^{+}-\frac{\hbar^{2}}{2 \mu}\left(k_{y}^{2}+k_{n}^{2}\right)} .
$$

This integral can be evaluated using standard contour integration techniques, ${ }^{36}$ giving us that

$$
\Gamma_{n}\left(y, y^{\prime} ; E\right)=\left\{\begin{array}{lll}
-i \frac{\mu}{\hbar^{2}} \frac{e^{i \kappa_{n}(E)\left|y-y^{\prime}\right|}}{\kappa_{n}(E)} & \text { if } & E>E_{n}, \\
-\frac{\mu}{\hbar^{2}} \frac{e^{-\kappa_{n}(E)\left|y-y^{\prime}\right|}}{\kappa_{n}(E)} & \text { if } & E<E_{n},
\end{array}\right.
$$

and

$$
\kappa_{n}(E)=\sqrt{\frac{2 \mu}{\hbar^{2}}\left|E-E_{n}\right|},
$$

where $E_{n}=\left(\hbar^{2} / 2 \mu\right) k_{n}^{2}$.

\section{Green's function, one impurity}

We introduce a single impurity modeled by an impurity potential $V(\vec{r})=\gamma \delta\left(\vec{r}-\vec{r}_{i}\right)$, where $\vec{r}_{i}$ is the position of the impurity and $\gamma$ the impurity strength. The perturbed propagator for an electron going from position $\vec{r}^{\prime}$ to $\vec{r}$ can then be written using the Dyson equation as ${ }^{37}$

$$
\begin{aligned}
G\left(\vec{r}, \vec{r}^{\prime} ; E\right)= & g\left(\vec{r}, \vec{r}^{\prime} ; E\right) \\
& +\int d \vec{r}^{\prime \prime} g\left(\vec{r}, \vec{r}^{\prime \prime} ; E\right) V\left(\vec{r}^{\prime \prime}\right) G\left(\vec{r}^{\prime \prime}, \vec{r}^{\prime} ; E\right) \\
= & g\left(\vec{r}, \vec{r}^{\prime} ; E\right)+g\left(\vec{r}, \vec{r}_{i} ; E\right) \gamma G\left(\vec{r}_{i}, \vec{r}^{\prime} ; E\right) \\
= & \sum_{n m}\left[g_{n}\left(\vec{r}, \vec{r}^{\prime} ; E\right) \delta_{n m}\right. \\
& +\underbrace{\left.\sum_{l} g_{n}\left(\vec{r}, \vec{r}_{i} ; E\right) \gamma G_{l m}\left(\vec{r}_{i}, \vec{r}^{\prime} ; E\right)\right]}_{=\tilde{G}_{n m}\left(\vec{r}, \vec{r}^{\prime} ; E\right)} \\
= & \sum_{n m} G_{n m}\left(\vec{r}, \vec{r}^{\prime} ; E\right) .
\end{aligned}
$$

The perturbed matrix element of the Dyson equation can be rewritten on the $T$ matrix form

$$
\tilde{G}_{n m}\left(\vec{r}, \vec{r}^{\prime} ; E\right)=g_{n}\left(\vec{r}, \vec{r}_{i} ; E\right) T\left(\vec{r}_{i} ; E\right) g_{m}\left(\vec{r}_{i}, \vec{r}^{\prime} ; E\right),
$$

where

$$
\begin{aligned}
T\left(\vec{r}_{i} ; E\right) & =\gamma+\gamma\left[\sum_{l} g_{l}\left(\vec{r}_{i}, \vec{r}_{i} ; E\right)\right] T\left(\vec{r}_{i} ; E\right) \\
& =\frac{\gamma}{1-\gamma \sum_{l} g_{l}\left(\vec{r}_{i}, \vec{r}_{i} ; E\right)} \\
& =\frac{1}{1 / \gamma+\sigma_{e}(E)+i \sigma_{p}(E)}
\end{aligned}
$$

and

$$
\sigma_{e / p}(E)=\frac{\mu}{\hbar^{2}} \sum_{l \in e / p} \frac{\chi_{l}^{2}\left(x_{i}\right)}{\kappa_{l}(E)} .
$$

Here, $e$ and $p$ are the sets of all evanescent $\left(E<E_{l}\right)$ and propagating $\left(E>E_{l}\right)$ modes. Inserting the above expression for $T\left(\vec{r}_{i} ; E\right)$ back into Eq. (A9) allows us to solve for $\tilde{G}_{n m}\left(\vec{r}, \vec{r}^{\prime} ; E\right)$ and consequently for $G_{n m}\left(\vec{r}, \vec{r}^{\prime} ; E\right)$.

\section{Fourier transformed density of states}

Once the perturbed propagator is known, the change in the LDOS due to scattering is given by

$$
\begin{aligned}
\tilde{\rho}(\vec{r} ; E) & =-\frac{1}{\pi} \sum_{n m} \operatorname{Im}\left[\tilde{G}_{n m}(\vec{r}, \vec{r} ; E)\right] \\
& =-\frac{1}{\pi} \sum_{n m} \mathcal{K}_{n m}(E) \tilde{\rho}_{n m}^{x}(x) \tilde{\rho}_{n m}^{y}(y ; E),
\end{aligned}
$$

where

$$
\mathcal{K}_{n m}(E)=\left(\frac{\mu}{\hbar^{2}}\right)^{2} \frac{1}{\left(1+\sigma_{e}(E)\right)^{2}+\sigma_{p}^{2}(E)} \frac{\chi_{n}\left(x_{i}\right) \chi_{m}\left(x_{i}\right)}{\kappa_{n}(E) \kappa_{m}(E)},
$$

$$
\tilde{\rho}_{n m}^{x}(x)=\chi_{n}(x) \chi_{m}(x)
$$


and

$$
\tilde{\rho}_{n m}^{y}(y ; E)= \begin{cases}-f_{s c}\left(\kappa_{n}(E), \kappa_{m}(E)\right) & \text { if } n, m \in p, \\ f_{c s}\left(\kappa_{n}(E), 0\right) e^{-\kappa_{m}(E)\left|y-y_{i}\right|} & \text { if } n \in p, m \in e, \\ f_{c s}\left(0, \kappa_{m}(E)\right) e^{-\kappa_{n}(E)\left|y-y_{i}\right|} & \text { if } n \in e, m \in p, \\ -\sigma_{p}(E) e^{-\left(\kappa_{n}(E)+\kappa_{m}(E)\right)\left|y-y_{i}\right|} & \text { if } n, m \in e,\end{cases}
$$

where

$$
\begin{aligned}
f_{c s}\left(\kappa_{1}, \kappa_{2}\right)= & \left(1+\sigma_{e}\right) \cos \left[\left(\kappa_{1}+\kappa_{2}\right)\left|y-y_{i}\right|\right] \\
& +\sigma_{p} \sin \left[\left(\kappa_{1}+\kappa_{2}\right)\left|y-y_{i}\right|\right]
\end{aligned}
$$

and

$$
\begin{aligned}
f_{s c}\left(\kappa_{1}, \kappa_{2}\right)= & \left(1+\sigma_{e}\right) \sin \left[\left(\kappa_{1}+\kappa_{2}\right)\left|y-y_{i}\right|\right] \\
& -\sigma_{p} \cos \left[\left(\kappa_{1}+\kappa_{2}\right)\left|y-y_{i}\right|\right] .
\end{aligned}
$$

When taking the Fourier transform of the scattering LDOS, we want to be able to resolve differences in $x$ momenta equal to or greater than $\pi / W$ (since this is the separation in $k_{x}$, or $k_{n}$, between two adjacent subbands). This requires us to integrate over the interval $[-W, W]$ and we extend the function $\tilde{\rho}_{n m}^{x}(x)$ such that it is even with respect to the origin. The Fourier transform is then defined as

$$
\begin{aligned}
\tilde{\mathcal{N}}_{n m}(\vec{q} ; E)= & \mathcal{K}_{n m}(E) \sum_{n^{\prime}=-\infty}^{\infty} \delta\left(\frac{q_{x}}{\pi}-\frac{n^{\prime}}{W}\right) \\
& \times \int_{-W}^{W} \frac{d x}{2 W} e^{-i q_{x} x} \tilde{\rho}_{n m}^{x}(x) \\
& \times \int_{-\infty}^{\infty} \frac{d y}{2 \pi} e^{-i q_{y} y} \tilde{\rho}_{n m}^{y}(y ; E),
\end{aligned}
$$

where the comb function fixes $q_{x}$ to multiples of $\pi / W$. The $x$ part of the Fourier integral is

$$
\begin{aligned}
& \int_{-W}^{W} \frac{d x}{2 W} e^{-i q_{x} x} \tilde{\rho}_{n m}^{x}(x) \\
& =\frac{1}{2 W}\left(\delta_{l,-n-m}+\delta_{l, n+m}-\delta_{l,-n+m}-\delta_{l, n-m}\right),
\end{aligned}
$$

independent of whether $n$ and $m$ are evanescent or propagating modes.

The $y$ part will depend on mode types. We have already shown what happens when $n, m \in p$. In addition, if $n, m \in e$ we get that

$$
\begin{aligned}
& \int_{-\infty}^{\infty} \frac{d y}{2 \pi} e^{-i q_{y} y} \tilde{\rho}_{n m}^{y}(y ; E) \\
& =-e^{-i q_{y} y_{i}} \frac{\sigma_{p}}{\pi} \frac{\kappa_{n}(E)+\kappa_{m}(E)}{q_{y}^{2}+\left(\kappa_{n}(E)+\kappa_{m}(E)\right)^{2}} .
\end{aligned}
$$

If $n \in p, m \in e$ then

$$
\begin{aligned}
\int_{-\infty}^{\infty} \frac{d y}{2 \pi} e^{-i q_{y} y} \tilde{\rho}_{n m}^{y}(y ; E)= & \frac{e^{-i q_{y} y_{i}}}{2 \pi}\left[S_{p e}^{y}\left(\kappa_{n}(E)-q_{y}, \kappa_{m}(E)\right)\right. \\
& \left.+S_{p e}^{y}\left(\kappa_{n}(E)+q_{y}, \kappa_{m}(E)\right)\right],
\end{aligned}
$$

where

$$
S_{p e}^{y}(a, b)=\frac{\left(1 / \gamma+\sigma_{e}(E)\right) b+\sigma_{p}(E) a}{b^{2}+a^{2}} .
$$

If $n \in e, m \in p$ we just need to interchange the $n$ and $m$ in the expression above.

\section{APPENDIX B: ARMCHAIR GRAPHENE NANORIBBON}

In this Appendix we first derive an analytic expression for the Green's function of an armchair nanoribbon with a single impurity. For the geometry, see Fig. 12(a). We then derive the Fourier transformed density of states.

\section{Unperturbed Green's function}

The first Brillouin zone (1BZ) of graphene contains one pair of inequivalent Dirac cones. It is necessary, however, to include three pairs of cones [see Fig. 12(b)] in order to incorporate all scattering events. The cones are located at $\vec{K}_{1}^{ \pm}=\left( \pm K_{x}, 0\right), \vec{K}_{2}^{ \pm}=\left( \pm K_{x} / 2, K_{y}\right)$, and $\vec{K}_{3}^{ \pm}=\left( \pm K_{x} / 2,-\right.$ $K_{y}$ ), where $K_{x}=4 \pi / 3 a$ and $K_{y}=2 \pi / 3 a_{0}$. The distance between two neighboring atoms is denoted $a_{0}$, while the lattice constant is denoted $a=\sqrt{3} a_{0}$. This gives us three sets $(d=1,2,3)$ of wave function spinors, ${ }^{38}$

$$
\vec{\Psi}_{d n}(\vec{r})=\left(\begin{array}{c}
\Psi_{d n}^{A}(y) \\
\Psi_{d n}^{B}(y)
\end{array}\right) \chi_{d n}(x),
$$

where the longitudinal wave function components are

$$
\begin{gathered}
\Psi_{d n}^{A}(y)=\lambda \frac{\left(k_{y}+i k_{d n}\right)}{\sqrt{k_{y}^{2}+k_{d n}^{2}}} e^{i\left(K_{d y}+k_{y}\right) y}, \\
\Psi_{d n}^{B}(y)=i e^{i\left(K_{d y}+k_{y}\right) y},
\end{gathered}
$$

and the transverse wave function is

$$
\chi_{d n}(x)=2 C \sin \left[\left(K_{d x}+k_{d n}\right) x\right]
$$

The integer number $n$ labels the quantized transverse momentum $k_{d n}=n \pi / W-K_{d x}$ in cone pair $d$. For each mode $n$, we have positive and negative energy subbands $\epsilon_{d n \lambda}\left(k_{y}\right)=$ $\lambda \hbar v_{f} \sqrt{k_{y}^{2}+\left(k_{d n}\right)^{2}}$ labeled by $\lambda= \pm 1$. The Fermi velocity is $v_{f}=3 a_{0} \tau / 2$, where $\tau$ gives the nearest-neighbor tightbinding hopping energy in Eq. (17). The wave functions have been normalized through a normalization constant $C=$ $\sqrt{1 / 4 W}$ found from the condition $\int_{0}^{W} d x\left|\chi_{d n}(x)\right|^{2}=1 / 2$. Thus, $\chi_{d n}(x)=\chi_{n}(x)=\sqrt{1 / W} \sin (n \pi / W x)$.
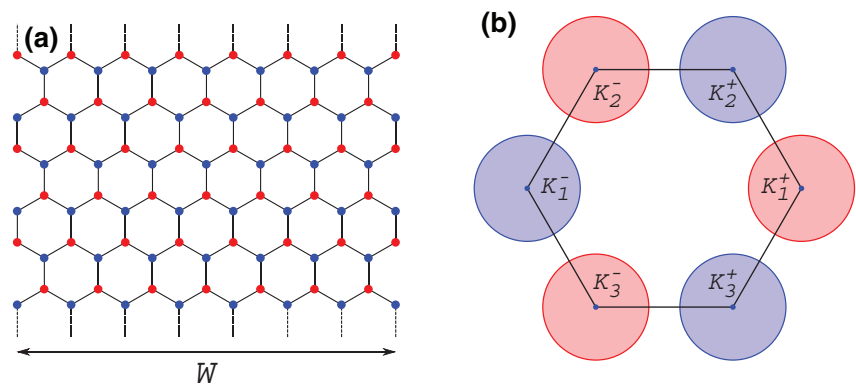

FIG. 12. (Color online) (a) The geometry of the armchair nanoribbon. (b) The first Brillouin zone with three sets of Dirac cones. 
The free propagator for band $n$ (in cone pair $d$ ) is computed as

$$
\begin{aligned}
\mathbf{g}_{d n}\left(\vec{r}, \vec{r}^{\prime}\right) & =\sum_{\lambda= \pm 1} \int_{-\infty}^{\infty} \frac{d k_{y}}{2 \pi} \frac{\vec{\Psi}_{d n}(\vec{r}) \vec{\Psi}_{d n}^{\dagger}\left(\vec{r}^{\prime}\right)}{E^{+}-\epsilon_{d n \lambda}\left(k_{y}\right)} \\
& =\chi_{n}(x) \chi_{n}\left(x^{\prime}\right)\left(\begin{array}{ll}
\Gamma_{d n}^{A A}\left(y, y^{\prime} ; E\right) & \Gamma_{d n}^{A B}\left(y, y^{\prime} ; E\right) \\
\Gamma_{d n}^{B A}\left(y, y^{\prime} ; E\right) & \Gamma_{d n}^{B B}\left(y, y^{\prime} ; E\right)
\end{array}\right),
\end{aligned}
$$

where

$$
\begin{aligned}
\Gamma_{d n}^{A A / B B}\left(y, y^{\prime} ; E\right)= & 2 E e^{i K_{d y}\left(y-y^{\prime}\right)} \\
& \times \int_{-\infty}^{\infty} \frac{d k_{y}}{2 \pi} \frac{e^{i k_{y}\left(y-y^{\prime}\right)}}{\left(E^{+}\right)^{2}-\left(\hbar v_{f}\right)^{2}\left(k_{y}^{2}+k_{d n}^{2}\right)},
\end{aligned}
$$

and

$$
\begin{aligned}
\Gamma_{d n}^{A B / B A}\left(y, y^{\prime} ; E\right)= & \mp 2 i \hbar v_{f} e^{i K_{d y}\left(y-y^{\prime}\right)} \\
& \times \int_{-\infty}^{\infty} \frac{d k_{y}}{2 \pi} \frac{\left(k_{y} \pm i k_{d n}\right) e^{i k_{y}\left(y-y^{\prime}\right)}}{\left(E^{+}\right)^{2}-\left(\hbar v_{f}\right)^{2}\left(k_{y}^{2}+k_{d n}^{2}\right)} .
\end{aligned}
$$

After contour integration, we find the final form to be

$$
\Gamma_{d n}^{A A / B B}\left(y, y^{\prime} ; E\right)=-i \frac{|E|}{\left(\hbar v_{f}\right)^{2}} e^{i K_{d y}\left(y-y^{\prime}\right)} \frac{e^{i \operatorname{sgn}(E) \kappa_{d n}(E)\left|y-y^{\prime}\right|}}{\kappa_{d n}(E)},
$$

and

$$
\begin{aligned}
\Gamma_{d n}^{A B / B A}\left(y, y^{\prime} ; E\right)= & -\frac{1}{\hbar v_{f}} e^{i K_{d y}\left(y-y^{\prime}\right)} \\
& \times\left[\frac{i \operatorname{sgn}(E) k_{d n}}{\kappa_{d n}(E)} \pm \operatorname{sgn}\left(y-y^{\prime}\right)\right] \\
& \times e^{i \operatorname{sgn}(E) \kappa_{d n}(E)\left|y-y^{\prime}\right|}
\end{aligned}
$$

where

$$
\kappa_{d n}=\sqrt{\left|\frac{E}{\left(\hbar v_{f}\right)^{2}}-k_{d n}^{2}\right|} .
$$

In the above formulas, we have assumed that $n$ is a propagating mode (e.g., $\left.\left|E /\left(\hbar v_{f}\right)\right|>\mid k_{d n}\right)$. If mode $n$ is evanescent
$\left(\left|E /\left(\hbar v_{f}\right)\right|<\left|k_{d n}\right|\right)$, we have to modify the longitudinal momentum so that $\kappa_{d n} \rightarrow i \operatorname{sgn}(E) \kappa_{d n}$.

\section{Green's function, one impurity}

For the graphene armchair ribbon, we select an impurity fully localized on the A sublattice,

$$
\mathbf{V}(\vec{r})=\gamma\left(\begin{array}{ll}
1 & 0 \\
0 & 0
\end{array}\right) \delta\left(\vec{r}-\vec{r}_{i}\right),
$$

where $\gamma$ is the impurity strength. The $T$-matrix equation is written in analogy to the 2DEG case, but for graphene it acquires a $2 \times 2$ matrix structure. For the potential in Eq. (B11), we get

$$
\begin{aligned}
\mathbf{T}\left(\vec{r}_{i} ; E\right)= & \gamma\left(\begin{array}{ll}
1 & 0 \\
0 & 0
\end{array}\right)+\gamma\left(\begin{array}{cc}
1 & 0 \\
0 & 0
\end{array}\right) \\
& \times\left[\sum_{d} \sum_{l} \mathbf{g}_{d l}\left(\vec{r}_{i}, \vec{r}_{i} ; E\right)\right] \mathbf{T}\left(\vec{r}_{i} ; E\right) \\
= & \frac{1}{1 / \gamma+\sigma_{e}(E)+i \sigma_{p}(E)}\left(\begin{array}{ll}
1 & 0 \\
0 & 0
\end{array}\right),
\end{aligned}
$$

where

$$
\sigma_{p}(E)=\frac{|E|}{\left(\hbar v_{f}\right)^{2}} \sum_{d} \sum_{l \in p} \frac{\chi_{l}^{2}\left(x_{i}\right)}{\kappa_{d l}(E)}
$$

and

$$
\sigma_{e}(E)=\frac{E}{\left(\hbar v_{f}\right)^{2}} \sum_{d} \sum_{l \in e} \frac{\chi_{l}^{2}\left(x_{i}\right)}{\kappa_{d l}(E)} .
$$

The letters $e$ and $p$ denote sets of evanescent and propagating modes, respectively. The Dyson equation for the Green's function can now be written as

$$
\mathbf{G}_{d n c m}\left(\vec{r}, \vec{r}^{\prime}\right)=\mathbf{g}_{d n}\left(\vec{r}, \vec{r}^{\prime}\right) \delta_{n m}+\tilde{\mathbf{G}}_{d n c m}\left(\vec{r}, \vec{r}^{\prime}\right),
$$

where

$$
\tilde{\mathbf{G}}_{d n c m}\left(\vec{r}, \vec{r}^{\prime} ; E\right)=\mathbf{g}_{d n}\left(\vec{r}, \vec{r}_{i} ; E\right) \mathbf{T}\left(\vec{r}_{i}, \vec{r}_{i} ; E\right) \mathbf{g}_{c m}\left(\vec{r}_{i}, \vec{r}^{\prime} ; E\right) .
$$

The scattering part $\tilde{\mathbf{G}}_{d n c m}\left(\vec{r}, \vec{r}^{\prime} ; E\right)$ takes the form

$$
\begin{aligned}
\tilde{\mathbf{G}}_{d n c m}\left(\vec{r}, \vec{r}^{\prime} ; E\right) & =\frac{1}{1 / \gamma+\sigma_{e}(E)+i \sigma_{p}(E)}\left(\begin{array}{ll}
g_{d n}^{A A}\left(\vec{r}, \vec{r}_{i} ; E\right) g_{c m}^{A A}\left(\vec{r}_{i}, \vec{r}^{\prime} ; E\right) & g_{d n}^{A A}\left(\vec{r}, \vec{r}_{i} ; E\right) g_{c m}^{A B}\left(\vec{r}_{i}, \vec{r}^{\prime} ; E\right) \\
g_{d n}^{B A}\left(\vec{r}, \vec{r}_{i} ; E\right) g_{c m}^{A A}\left(\vec{r}_{i}, \vec{r}^{\prime} ; E\right) & g_{d n}^{B A}\left(\vec{r}, \vec{r}_{i} ; E\right) g_{c m}^{A B}\left(\vec{r}_{i}, \vec{r}^{\prime} ; E\right)
\end{array}\right) \\
& =\frac{\chi_{n}(x) \chi_{n}\left(x_{i}\right) \chi_{m}\left(x_{i}\right) \chi_{m}\left(x^{\prime}\right)}{1 / \gamma+\sigma_{e}(E)+i \sigma_{p}(E)}\left(\begin{array}{cc}
\Gamma_{d n}^{A A}\left(y, y_{i} ; E\right) \Gamma_{c m}^{A A}\left(y_{i}, y^{\prime} ; E\right) & \Gamma_{d n}^{A A}\left(y, y_{i} ; E\right) \Gamma_{c m}^{A B}\left(y_{i}, y^{\prime} ; E\right) \\
\Gamma_{d n}^{B A}\left(y, y_{i} ; E\right) \Gamma_{c m}^{A A}\left(y_{i}, y^{\prime} ; E\right) & \Gamma_{d n}^{B A}\left(y, y_{i} ; E\right) \Gamma_{c m}^{A B}\left(y_{i}, y^{\prime} ; E\right)
\end{array}\right)
\end{aligned}
$$

For the computation of the LDOS, we need the two diagonal components. Their explicit forms $(n, m \in p)$ are

$$
\begin{aligned}
\tilde{G}_{d n c m}^{A A}\left(\vec{r}, \vec{r}^{\prime} ; E\right)= & -\frac{1}{1 / \gamma+\sigma_{e}(E)+i \sigma_{p}(E)}\left(\frac{|E|}{\left(\hbar v_{f}\right)^{2}}\right)^{2} \chi_{n}(x) \chi_{n}\left(x_{i}\right) \chi_{m}\left(x_{i}\right) \chi_{m}\left(x^{\prime}\right) \\
& \times e^{i K_{d y}\left(y-y_{i}\right)} e^{i K_{c y}\left(y_{i}-y^{\prime}\right)} \frac{e^{i \operatorname{sgn}(E)\left(\kappa_{d n}(E)\left|y-y_{i}\right|+\kappa_{c m}(E)\left|y_{i}-y^{\prime}\right|\right)}}{\kappa_{d n}(E) \kappa_{c m}(E)}
\end{aligned}
$$


and

$$
\begin{aligned}
\tilde{G}_{d n c m}^{B B}\left(\vec{r}, \vec{r}^{\prime} ; E\right)= & \frac{1}{1 / \gamma+\sigma_{e}(E)+i \sigma_{p}(E)} \frac{1}{\left(\hbar v_{f}\right)^{2}} \chi_{n}(x) \chi_{n}\left(x_{i}\right) \chi_{m}\left(x_{i}\right) \chi_{m}\left(x^{\prime}\right) e^{i K_{d y}\left(y-y_{i}\right)} e^{i K_{c y}\left(y_{i}-y^{\prime}\right)} e^{i \operatorname{sgn}(E)\left(\kappa_{d n}(E)\left|y-y_{i}\right|+\kappa_{c m}(E)\left|y_{i}-y^{\prime}\right|\right)} \\
& \times\left[\frac{i \operatorname{sgn}(E) k_{d n}}{\kappa_{d n}(E)}-\operatorname{sgn}\left(y-y_{i}\right)\right]\left[\frac{i \operatorname{sgn}(E) k_{c m}}{\kappa_{c m}(E)}+\operatorname{sgn}\left(y_{i}-y^{\prime}\right)\right] .
\end{aligned}
$$

\section{Density of states}

The scattering correction to the LDOS can be computed separately for the two sublattices, and is given by

$$
\begin{aligned}
\tilde{\rho}_{A / B}(\vec{r} ; E) & =-\frac{1}{\pi} \sum_{d c} \sum_{n m} \operatorname{Im}\left[\tilde{G}_{d n c m}^{A A / B B}(\vec{r}, \vec{r} ; E)\right] \\
& =-\frac{1}{\pi} \sum_{d c} \sum_{n m} \mathcal{K}_{d n c m}^{A / B}(E) \tilde{\rho}_{n m}^{x}(x) \tilde{\rho}_{d n c m}^{(A / B) y}(y ; E),
\end{aligned}
$$

where $\tilde{\rho}_{n m}^{x}(x)=\chi_{n}(x) \chi_{m}(x)$. The $\mathrm{A} / \mathrm{B}$ sublattice corrections are found by substituting Eq. (B18) and Eq. (B19), respectively, in Eq. (B20). The results are very similar (the A correction being almost identical) to the 2DEG case, and for $n, m \in p$ we find that

$$
\begin{aligned}
\mathcal{K}_{d n c m}^{A}(E)= & \frac{1}{\left(1 / \gamma+\sigma_{e}(E)\right)^{2}+\sigma_{p}^{2}(E)}\left(\frac{|E|}{\left(\hbar v_{f}\right)^{2}}\right)^{2} \\
& \times \frac{\chi_{n}\left(\chi_{i}\right) \chi_{m}\left(x_{i}\right)}{\kappa_{d n}(E) \kappa_{c m}(E)}, \\
\mathcal{K}_{d n c m}^{B}(E)=-\left(\hbar v_{f} /|E|\right)^{2} \mathcal{K}_{d n c m}^{A}(E), & \\
\tilde{\rho}_{d n c m}^{A y}(y ; E)= & \sigma_{p}(E) F_{d n c m}^{c}\left(y-y_{i} ; E\right) \\
& -\left(1 / \gamma+\sigma_{e}(E)\right) F_{d n c m}^{s}\left(y-y_{i} ; E\right),
\end{aligned}
$$

and

$$
\begin{aligned}
\tilde{\rho}_{d n c m}^{B y}(y ; E)= & \left\{\sigma_{p}(E)\left(-k_{n} k_{m}+\kappa_{n}(E) \kappa_{m}(E)\right)\right. \\
& +\left(1 / \gamma+\sigma_{e}(E)\right) \operatorname{sgn}(E) \operatorname{sgn}\left(y-y_{i}\right)\left(k_{n} \kappa_{m}(E)\right. \\
& \left.\left.+k_{m} \kappa_{n}(E)\right)\right\} F_{d n c m}^{c}\left(y-y_{i} ; E\right) \\
& +\left\{\sigma _ { p } ( E ) \operatorname { s g n } ( E ) \operatorname { s g n } ( y - y _ { i } ) \left(k_{n} \kappa_{m}(E)\right.\right. \\
& \left.+k_{m} \kappa_{n}(E)\right)-\left(1 / \gamma+\sigma_{e}(E)\right) \operatorname{sgn}(E) \\
& \left.\times \operatorname{sgn}\left(y-y_{i}\right)\left(k_{n} \kappa_{m}(E)+k_{m} \kappa_{n}(E)\right)\right\} \\
& \times F_{d n c m}^{s}\left(y-y_{i} ; E\right), \quad(\mathrm{B} 24)
\end{aligned}
$$

where

$$
\begin{aligned}
F_{d n c m}^{c}(y ; E)= & \cos \left[\operatorname{sgn}(E)\left(\kappa_{d n}(E)+\kappa_{c m}(E)\right)|y|\right. \\
& \left.+\left(K_{d y}-K_{c y}\right) y\right]
\end{aligned}
$$

and

$$
\begin{aligned}
F_{d n c m}^{s}(y ; E)= & \sin \left[\operatorname{sgn}(E)\left(\kappa_{d n}(E)+\kappa_{c m}(E)\right)|y|\right. \\
& \left.+\left(K_{d y}-K_{c y}\right) y\right] .
\end{aligned}
$$

The Fourier transform of each component is carried out exactly as for the 2DEG, using Eq. (A18), and the results for the AGNR are shown in Sec. IV.
${ }^{1}$ A. H. Castro Neto, F. Guinea, N. M. R. Peres, K. S. Novoselov, and A. K. Geim, Rev. Mod. Phys. 81, 109 (2009).

${ }^{2}$ F. Schedin, A. K. Geim, S. V. Morozov, E. W. Hill, P. Blake, M. I. Katsnelson, and K. S. Novoselov, Nat. Mater. 6, 652 (2007).

${ }^{3}$ V. W. Brar, R. Decker, H.-M. Solowan, Y. Wang, L. Maserati, K. T. Chan, H. Lee, Ç. O. Girit, A. Zettl, S. G. Louie, M. L. Cohen, and M. F. Crommie, Nat. Phys. 7, 43 (2011).

${ }^{4}$ N. Peres, Rev. Mod. Phys. 82, 2673 (2010).

${ }^{5}$ M. R. Connolly and C. G. Smith, Philos. Trans. R. Soc., A 368, 5379 (2010).

${ }^{6}$ A. Deshpande and B. J. LeRoy, Phys. E (Amsterdam, Neth.) 44, 743 (2012).

${ }^{7}$ G. M. Rutter, J. N. Crain, N. P. Guisinger, T. Li, P. N. First, and J. A. Stroscio, Science (New York, NY) 317, 219 (2007).

${ }^{8}$ P. Mallet, F. Varchon, C. Naud, L. Magaud, C. Berger, and J.-Y. Veuillen, Phys. Rev. B 76, 041403 (2007).

${ }^{9}$ J. Xue, J. Sanchez-Yamagishi, K. Watanabe, T. Taniguchi, P. JarilloHerrero, and Brian J. LeRoy, Phys. Rev. Lett. 108, 016801 (2012). ${ }^{10}$ Y. Zhang, V. W. Brar, C. Girit, A. Zettl, and M. F. Crommie, Nat. Phys. 5, 722 (2009).
${ }^{11}$ S.-H. Ji, J. B. Hannon, R. M. Tromp, V. Perebeinos, J. Tersoff, and F. M. Ross, Nat. Mater. 11, 114 (2011).

${ }^{12}$ W. Wang, K. Munakata, M. Rozler, and M. R. Beasley, arXiv:1211.6088.

${ }^{13}$ F. Giannazzo, I. Deretzis, A. La Magna, F. Roccaforte, and R. Yakimova, Phys. Rev. B 86, 235422 (2012).

${ }^{14}$ M. Y. Han, B. Özyilmaz, Y. Zhang, and P. Kim, Phys. Rev. Lett. 98, 206805 (2007)

${ }^{15}$ L. P. Biro and P. Lambin, Carbon 48, 2677 (2010).

${ }^{16}$ L. C. Campos, V. R. Manfrinato, J. D. Sanchez-Yamagishi, J. Kong, and P. Jarillo-Herrero, Nano Lett. 9, 2600 (2009).

${ }^{17}$ Ç. Ö. Girit, J. C. Meyer, R. Erni, M. D. Rossell, C. Kisielowski, L. Yang, C. H. Park, M. F. Crommie, M. L. Cohen, and S. G. Louie, Science (New York, NY) 323, 1705 (2009).

${ }^{18}$ J. Cai, P. Ruffieux, R. Jaafar, M. Bieri, T. Braun, S. Blankenburg, M. Muoth, A. P. Seitsonen, M. Saleh, X. Feng, K. Müllen, and R. Fasel, Nature (London) 466, 470 (2010).

${ }^{19}$ X. Li, X. Wang, L. Zhang, S. Lee, and H. Dai, Science (New York, NY) 319, 1229 (2008).

${ }^{20}$ M. Fujita, K. Wakabayashi, K. Nakada, and K. Kusakabe, J. Phys. Soc. Jpn. 65, 1920 (1996). 
${ }^{21}$ K. Nakada, M. Fujita, G. Dresselhaus, and M. S. Dresselhaus, Phys. Rev. B 54, 17954 (1996)

${ }^{22}$ C. Tao, L. Jiao, O. V. Yazyev, Y.-C. Chen, J. Feng, X. Zhang, R. B. Capaz, J. M. Tour, A. Zettl, S. G. Louie, H. Dai, and M. F. Crommie, Nat. Phys. 7, 616 (2011).

${ }^{23}$ J. J. Palacios, J. Fernandez-Rossier, L. Brey, and H. A. Fertig, Semicond. Sci. Technol. 25, 033003 (2010).

${ }^{24}$ H. Raza (editor), Graphene Nanoelectronics; Metrology, Synthesis, Properties and Applications (Springer-Verlag, Berlin, Heidelberg, 2012).

${ }^{25}$ T. Pereg-Barnea and A. H. MacDonald, Phys. Rev. B 78, 014201 (2008).

${ }^{26}$ C. Bena, Phys. Rev. Lett. 100, 076601 (2008).

${ }^{27}$ L. Petersen, P. Hofmann, E. W. Plummer, and F. Besenbacher, J. Electron Spectrosc. Relat. Phenom. 109, 97 (2000).

${ }^{28}$ A. V. Balatsky, I. Vekhter, and J.-X. Zhu, Rev. Mod. Phys. 78, 373 (2006).
${ }^{29}$ D. Boese, M. Lischka, and L. E. Reichl, Phys. Rev. B 61, 5632 (2000).

${ }^{30}$ K. Kazymyrenko and X. Waintal, Phys. Rev. B 77, 115119 (2008).

${ }^{31}$ K. Wakabayashi, Y. Takane, M. Yamamoto, and M. Sigrist, New J. Phys. 11, 095016 (2009).

${ }^{32}$ K. Wakabayashi, Y. Takane, and M. Sigrist, Phys. Rev. Lett. 99, 036601 (2007).

${ }^{33}$ H. P. Dahal, A. V. Balatsky, and J.-X. Zhu, Phys. Rev. B 77, 115114 (2008).

${ }^{34}$ T. O. Wehling, M. I. Katsnelson, and A. I. Lichtenstein, Phys. Rev. B 80, 085428 (2009).

${ }^{35}$ S. Ihnatsenka and G. Kirczenow, Phys. Rev. B 83, 245442 (2011).

${ }^{36}$ E. N. Economou, Green's Functions in Quantum Physics, 3rd Ed. (Springer-Verlag, Berlin, 2006).

${ }^{37}$ P. Bagwell, J. Phys.: Condens. Matter 2, 6179 (1990).

${ }^{38}$ L. Brey and H. A. Fertig, Phys. Rev. B 73, 235411 (2006). 